TRABAJO FIN DE MÁSTER

\title{
PROPUESTA DE UNA NUEVA METODOLOGÍA PARA EL CONTROL DE RESIDUOS DE BENZIMIDAZOLES MEDIANTE CROMATOGRAFÍA DE LÍQUIDOS CAPILAR
}

MÁSTER EN “CIENCIAS Y TECNOLOGÍAS QUÍMICAS, KHEMIA"

UNIVERSIDAD DE GRANADA

TRABAJO FIN DE MÁSTER

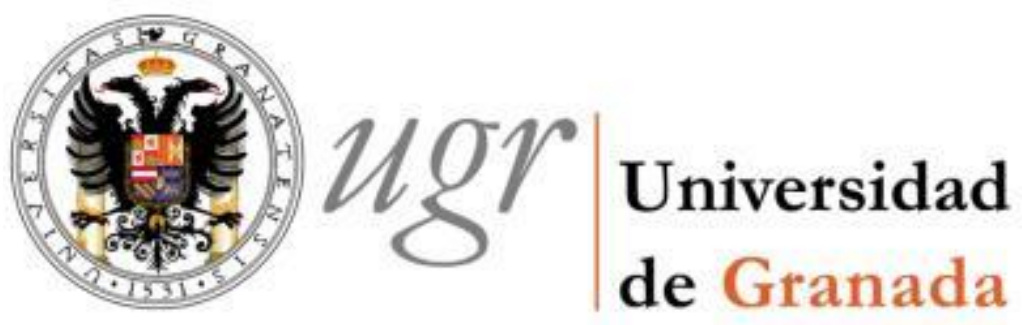

MARÍA DOLORES ESCOLANO GÁLVEZ 
El Trabajo Fin de Máster (TFM) que se presenta en este documento es realizado en el grupo de investigación "Calidad en Química Analítica, Alimentaria, Ambiental y Clínica" (FQM-302) del Departamento de Química Analítica de la Facultad de Ciencias de la Universidad de Granada, tutelado por los Dres. Ana M. García Campaña, catedrática de la Universidad de Granada y Francisco J. Lara Vargas, investigador postdoctoral del Programa Talent Hub. 
PROPUESTA DE UNA NUEVA METODOLOGÍA PARA EL CONTROL DE RESIDUOS DE BENZIMIDAZOLES MEDIANTE CROMATOGRAFÍA DE LÍQUIDOS CAPILAR

TRABAJO FIN DE MÁSTER

\section{AGRADECIMIENTOS}

A todas aquellas personas que de un modo $u$ otro me han ayudado y aconsejado para realizar este TFM. 
TRABAJO FIN DE MÁSTER

ÍNDICE

1. PRESENTACIÓN

\section{BLOQUE I}

\section{BLOQUE II}

1. INTRODUCCIÓN

1.1. JUSTIFICACIÓN DEL ESTUDIO

1.2. LEGISLACIÓN DEL CONTROL DE RESIDUOS DE BENZIMIDAZOLES

1.3. OBJETIVO DEL ESTUDIO

1.4. ANTECEDENTES

1.4.1. Características de los benzimidazoles

1.4.2. Cromatografía de liquidos capilar

1.4.3. Tratamientos de muestra

2. OBJETIVOS ESPECÍFICOS

3. METODOLOGÍA: MATERIAL Y MÉTODOS

3.1. TIPO DE ESTUDIO Y DE DISEÑO

3.2. RECURSOS

3.3. FASES DE ESTUDIO

4. RESULTADOS Y DISCUSIÓN

4.1. CONDICIONES INICIALES 22

4.1.1. Gradiente $\quad 22$

4.1.2. Fase móvil $\quad 23$

4.2. PARÁMETROS A OPTIMIZAR 23

4.2.1. Características de la disolución $\quad 23$

4.2.2. Flujo de la fase móvil $\quad 25$

4.2.3. Temperatura de la columna $\quad \mathbf{2 8}$

4.2.4. Disolvente de la muestra $\quad 30$

4.2.5. Volumen de inyección $\quad 32$

4.3. CURVA DE CALIBRADO 34

4.3.1. Parámetros estadísticos $\quad 37$

4.4. TRATAMIENTO DE MUESTRA $\mathbf{4 0}$

4.5. VALIDACIÓN DEL MÉTODO

5. CONCLUSIONES

46

6. BIBLIOGRAFÍA 
TRABAJO FIN DE MÁSTER

\section{BLOQUE I}

\section{PRESENTACIÓN}

Tras finalizar Licenciatura en Químicas por la Universidad de Alicante, me decidí a realizar el Máster Oficial en Ciencias y Tecnologías Químicas, Khemia con el fin de aumentar y mejorar mis conocimientos en diversos ámbitos de Química. Si bien durante la carrera se hace un estudio exhaustivo y profundo de conocimientos teóricos en las principales ramas de la química, la falta de contacto con equipos de laboratorio es evidente. Tras cinco años de carrera, la duración de prácticas en los distintos departamentos no excedía de más de una semana; a excepción de aquellas asignaturas que se impartían directamente en el laboratorio, pero en las que la utilización de equipos más sofisticados que no estaba al alcance del alumnado.

Por ello la decisión de realizar este máster tenía como objetivo, no sólo recordar y ampliar conocimientos teóricos, sino también a través de las asignaturas y sus prácticas poder tomar contacto con un mayor número de equipos que los investigadores utilizaran de forma rutinaria para sus proyectos de investigación. Esta sería la causa por lo que se eligió el itinerario de Metodología e Instrumentación y dentro de este itinerario la elección de las asignaturas tales como:

- Herramientas para el Análisis de Procesos Químicos.

- Espectrometría de Rayos X

- Nanomateriales

- Microscopía Analítica

- Caracterización de la Superficie de Sólidos

- Caracterización de Compuestos de Coordinación

- Ingeniería y Caracterización de Proteínas

- Quimiometría

- Plataformas Instrumentales Avanzadas para el Análisis Químico

- Gestión de la Instrumentación, Calibración y Verificaciones

En todas ellas, además de los conocimientos teóricos, se han visto multitud de equipos incluyendo en algunos casos su utilización. En la mayoría de estas asignaturas se ha visitado el Centro de Instrumentación Científica (CIC) de la Universidad de Granada (UGR), donde se han visto equipos como:

- Difracción de Rayos X de polvo o de cristal único.

- Espectrometría de Absorción Atómica

- Resonancia Magnética Nuclear

- Microsonda lónica

- Microscopía de Barrido Láser Confoca

Estos son equipos de gran potencial y valor económico, que son manejados por personas cualificadas en los mismos. El profesorado ha explicado previamente el fundamento y la utilización de ellos para verlos a posteriori; en ocasiones hasta analizando muestras en la visita al CIC.

El objetivo a la hora de realizar el Máster, incluyendo el itinerario elegido y una vez cursadas las asignaturas, la elección de mi Trabajo Fin de Máster (TFM) estuvo orientado a la utilización de la técnica separativa de cromatografía. 


\section{TRABAJO FIN DE MÁSTER}

El Trabajo Fin de Máster se ha llevado a cabo en el Departamento de Química Analítica dentro del Grupo de Investigación Calidad en Química Analítica alimentaria, ambiental y clínica (FQM 302) dirigido por la Dra. Ana M. García Campaña. Ha sido autorizado por ella junto con el Dr. Francisco Jesús Lara Vargas. Las líneas de investigación que llevan a cabo:

$>$ Calidad y seguridad en los alimentos

- Análisis de residuos de pesticidas en frutas y verduras

- Análisis de residuos de fármacos (principalmente antibióticos) en los alimentos de origen animal (leche, carne, huevos, etc.)

- Desarrollo de métodos analíticos para la detección de micotoxinas en los alimentos y bebidas.

$>$ Control de la calidad ambiental

- Desarrollo de métodos analíticos para la detección de residuos de plaguicidas y sus productos de degradación en muestras de suelos, lodos y agua.

- Desarrollo de métodos analíticos para el análisis de contaminantes orgánicos emergentes (productos farmacéuticos, antibióticos, hormonas, etc.) en las muestras de agua naturales y las muestras de aguas residuales.

$>$ Control de calidad de los productos farmacéuticos y monitorización terapéutica en fluidos biológicos

- Propuesta de métodos rápidos para el control de calidad de los compuestos activos en medicamentos.

- Estudio de productos de degradación, la detección de impurezas y la separación de enantiómeros en fármacos.

- Análisis de fármacos en fluidos biológicos (orina, suero, plasma) para el ajuste de la dosis terapéutica.

$>$ Análisis de drogas de abuso en muestras de interés en los laboratorios forenses

- Desarrollo de metodologías alternativas que emplean técnicas miniaturizado para la determinación de las drogas de abuso.

- Estudio de las diferentes posibilidades técnicas de pre-concentración y extracción para el análisis de drogas de abuso en numerosas muestras de interés forense (sangre, suero, orina, pelo, etc.).

La principal prioridad del equipo es la propuesta de métodos analíticos nuevos, rápidos, sensibles y fiables, basado en técnicas miniaturizadas, compatibles con el medio ambiente, y con la sensibilidad requerida por los límites legales establecidos por la UE en diferentes campos.

Este grupo de investigación posee gran variedad de equipos, entre los que se encuentra cromatógrafos de líquidos (LC) acoplados a diversos sistemas de detección tales como UV-visible, fluorescencia o espectrometría masas (MS). También disponen de equipos para realizar electroforesis capilar (CE) acoplados a similares sistemas de detección. 
Entre los proyectos de investigación financiados que desarrollan en la actualidad se encuentra:

- Agentes químicos de riesgo en nutracéuticos: propuestas analíticas avanzadas para la determinación de micotoxinas y plaguicidas.

- Aplicación de técnicas de espectrometría de masas emergente para la selección de alimentos para residuos y contaminantes.

- Metodologías avanzadas de análisis y tratamiento de la muestra en base a la miniaturización y la química verde para el control de residuos múltiples de agentes químicos peligrosos en los alimentos y alimentos de origen animal infantiles

En el TFM realizado nos centraremos en Calidad y seguridad en los alimentos, en particular en busca de aplicaciones prácticas de cromatografía de líquidos.

Para terminar con el bloque de presentación debemos hablar de las competencias adquiridas durante la realización de Máster en Ciencias y Tecnologías Químicas (KHEMIA).

Dentro de las competencias básicas, destacan:

- CB06: Poseer y comprender conocimientos que aporten una base u oportunidad de ser originales en el desarrollo y/o aplicación de ideas, a menudo en un contexto de investigación.

- CB07: Que los estudiantes sepan aplicar los conocimientos adquiridos y su capacidad de resolución de problemas en entornos nuevos o poco conocidos dentro de contextos más amplios (o multidisciplinares) relacionados con su área de estudio.

- CB08: Que los estudiantes sean capaces de integrar conocimientos y enfrentarse a la complejidad de formular juicios a partir de una información que, siendo incompleta o limitada, incluya reflexiones sobre las responsabilidades sociales y éticas vinculadas a la aplicación de sus conocimientos y juicios.

- CB09: Que los estudiantes sepan comunicar sus conclusiones, y los conocimientos y razones últimas que las sustentan, a públicos especializados y no especializados de un modo claro y sin ambigüedades.

- CB10: Que los estudiantes posean las habilidades de aprendizaje que les permitan continuar estudiando de un modo que habrá de ser en gran medida autodirigido o autónomo.

- CG01: Que los estudiantes sepan asumir las responsabilidades adecuadas en lo que respecta al desarrollo de conocimientos y/o prácticas profesionales.

Podemos afirmar que se ha conseguido la adquisición de todas ellas en mayor o menor medida, ya que en algunas asignaturas ha habido una interacción profesor-alumno a la hora de exponer nuestros conocimientos en distintas materias y sacar conclusiones de forma individual tras la información adquirida. La realización de ejercicios, trabajos o exposiciones nos permite una práctica de búsqueda de información; la utilización de programas para la realización de ejercicios permite desarrollar nuestra capacidad creativa así como obtener conclusiones propias tras el trabajo realizado. Debemos añadir la utilización de diferentes software que permiten un estudio de la información dada por los equipos de análisis. 


\section{TRABAJO FIN DE MÁSTER}

En cuanto a las competencias transversales destacan:

- CT02: Capacidad de gestión del tiempo (referida a su organización y planificación).

- CT4: Conocimiento y uso de las tecnologías de la información y comunicación (TIC) y de los recursos informáticos (programas, base de datos, etc.) relativos al ámbito de estudio para adquirir, gestionar y comunicar la información.

- CT06: Trabajo en equipo.

- CT07: Sensibilidad hacia temas medioambientales.

Las competencias transversales a lo largo del desarrollo del Trabajo Fin de Máster (TFM) se adquieren de manera conjunta, ya que es necesaria una planificación del tiempo para poder desarrollarlo adecuadamente; la utilización tanto de programas, base de datos y software específico al equipo utilizado es esencial para el trabajo de investigación. En cuanto a temas medioambientales nos referimos, se busca la mínima utilización de reactivos para un menor deshecho de los mismos; y el trabajo en equipo no se puede cuestionar en base al trabajo de investigación que se realiza como alumna de Máster 


\section{BLOQUE II}

\section{INTRODUCCIÓN}

\subsection{JUSTIFICACIÓN DEL ESTUDIO}

Durante décadas, el ser humano ha sembrado y cultivado los campos para su alimentación y ha criado y alimentado a los animales que más tarde iba a utilizar como sustento. La industrialización y evolución de la sociedad ha provocado que los métodos en la agricultura y crianza de animales, incluyendo los alimentos para su crecimiento y el tratamiento de los mismos sea en ocasiones abusivo en lo que respecta a la rapidez de crecimiento de lo sembrado, o a la cantidad de sustancias dirigidas para obtener animales con mejores características. La alimentación ha sido una de las causas principales de la aparición y desarrollo de muchas enfermedades que hoy día sufren las personas, por tanto los animales que ingerimos son una causa directa de las mismas. Es por ello que se ha mostrado un gran interés en los últimos años por el estudio y preocupación sobre sustancias que pueden contener los animales que más tardes son utilizados como fuente de alimentación de los seres humanos.

De acuerdo al nombre del TFM(PROPUESTA DE UNA NUEVA METODOLOGÍA PARA EL CONTROL DE RESIDUOS DE BENZIMIDAZOLES MEDIANTE CROMATOGRAFÍA DE LÍQUIDOS CAPILAR) y realizando la investigación, como ya sabemos en el Departamento de Química Analítica dentro del Grupo de Investigación: Calidad en Química Analítica alimentaria, ambiental y clínica, debemos justificar el estudio en función de la familia de compuestos que determinamos mediante Cromatografía de líquidos capilar ( $\mu \mathrm{HPLC})$. La familia de compuestos con los que se lleva a cabo el estudio son los benzimidazoles.

Los benzimidazoles son medicamentos veterinarios utilizados para la prevención y tratamiento de infecciones parasitarias en la agricultura y la acuicultura. Algunos benzimidazoles también han encontrado aplicaciones como fungicidas pre o post-cosecha para el control de una amplia gama de hongos que afectan a los cultivos de campo, frutas y verduras almacenadas. Tiabendazol (TBZ) fue el primer benzimidazol comercializado hace más de 40 años [1]. Se ha utilizado ampliamente para el control de nematodos gastrointestinales, nematodos pulmonares y como un agente fungicida. Después de su introducción, una serie de benzimidazoles son alternativas, y ofrecen una actividad similar, tales como mebendazol (MBZ)[2] y oxibendazol (OXI)[3].

Los benzimidazoles poseen sulfuro y sulfóxido, grupos funcionales que se introdujeron posteriormente, que ofrece un espectro más amplio de actividad y eficacia mejorada. Albendazol (ABZ)[4] y fenbendazol (FBZ) [5] fueron de los primeros benzimidazoles utilizados con éxito en el tratamiento de todas las etapas de crecimiento de los nematodos gastrointestinales. Se pueden usar también en el tratamiento de parásitos pulmonares, tenías y etapas adultas de fasciola hepática. El benzimidazol, triclabendazol (TCB) se introdujo más tarde como un agente antihelmíntico para el tratamiento de todas las etapas de fasciola hepática, pero es ineficaz contra los nematodos [6]. La baja solubilidad de los sulfuros y sulfóxidos de benzimidazol conduce a su baja absorción desde el intestino, dando como resultado una baja biodisponibilidad [7,8]. Netobimina (NETO) [9] y febantel (FEB) [10], que son los profármacos de ABZ y FBZ, respectivamente, tienen una mayor solubilidad en agua con una mejor absorción y biodisponibilidad aumentada.

En el ganado vacuno y las ovejas tratadas con $A B Z$, albendazol sulfóxido ( $A B Z-S O$ ) se ha demostrado que $\mathrm{ABZ}-\mathrm{SO}$ o Albendazolsulfona $\left(\mathrm{ABZ}-\mathrm{SO}_{2}\right)$ son los residuos más probables que se encuentre en el caso de una violación del Límite Máximo de Residuos (LMR). Aminoalbendazolsulfona $\left(\mathrm{ABZ}-\mathrm{NH}_{2}-\mathrm{SO}_{2}\right)$ 
es quizás el residuo más persistente en el tejido, pero en general se produce a niveles por debajo del LMR. Hay muy poca información disponible sobre la presencia de residuos individuales FBZ en los tejidos [11]. En ganado vacuno tratado con FEB o FBZ era el residuo principal que se encuentra en los períodos de retiro anticipado, con menores niveles de febendacolsulfona (FBZ-SO 2 ).

En el ganado vacuno, caprino y bovino tratado con TBZ, el fármaco original ha demostrado ser el residuo principal que se encuentra en el hígado después del tratamiento. El metabolito 5-hidroxitiabendazol (5$\mathrm{OH}-\mathrm{TBZ}$ ) se produce sólo en niveles bajos en estas especies, pero se demostró que era el principal metabolito en aves de corral en los períodos de retirada anticipada. Hay muy poca información disponible sobre la presencia de residuos en los tejidos individuales de TCB. Sin embargo, los datos fármacocinéticos indican que el triclabendazol sulfóxido (TCB-SO) y el triclabendazolsulfona (TCB-SO $)_{2}$ serían los residuos más probables.

A continuación mostramos una tabla con los 21 benzimidazoles de los que partimos para llevar a cabo el estudio del método analítico desarrollado en este TFM, donde se muestra su estructura y otras características:

Tabla 1: Descripción de los veintiún Benzimidazoles de los que parte nuestro estudio.

\begin{tabular}{|c|c|c|c|}
\hline NOMBRE & ESTRUCTURA & pKa & $\begin{array}{c}\mathrm{Pm} \\
(\mathrm{g} / \mathrm{mol})\end{array}$ \\
\hline $\begin{array}{l}\text { ALBENDAZOL (ABZ):[5-(propiltio)- } \\
\text { 1H-benzimidazol-2-il]-, metilester } \\
(9 \mathrm{Cl})\end{array}$ & & 10.72 & 265.33 \\
\hline $\begin{array}{l}\text { ALBENDAZOL SULFONA (ABZ- } \\
\text { SO2): } N \text {-[6-(propilsulfonil)-1H- } \\
\text { benzimidazol-2-il]-, metilester }\end{array}$ & & 10.11 & 297.33 \\
\hline $\begin{array}{l}\text { ALBENDAZOL SULFOXIDO (ABZ- } \\
\text { SO): } N \text {-[6-(propilsulfinil)-1H- } \\
\text { benzimidazol-2-il]-, metilester }\end{array}$ & & 10.05 & 281.33 \\
\hline $\begin{array}{l}\text { FEBENDAZOL (FBZ): [Metil 5- } \\
\text { (feniltio)-2-benzimidazolcarbamato] }\end{array}$ & & 11.78 & 299.35 \\
\hline $\begin{array}{l}\text { FEBENDAZOL SULFONA (FBZ- } \\
\text { SO2): } N \text {-[6-(fenilsulfonil)-1H- } \\
\text { benzimidazol-2-il]-, metilester }\end{array}$ & & 10.14 & 331.35 \\
\hline $\begin{array}{c}\text { FEBENDAZOL SULFOXIDO (FBZ- } \\
\text { SO): } N \text {-[6-(fenilsulfonil)-1 } \mathrm{H} \text { - } \\
\text { benzimidazol-2-il]-, metilester }\end{array}$ & & 7.8 & 315.35 \\
\hline
\end{tabular}


PROPUESTA DE UNA NUEVA METODOLOGÍA PARA EL CONTROL DE RESIDUOS DE BENZIMIDAZOLES MEDIANTE CROMATOGRAFÍA DE LÍQUIDOS CAPILAR

TRABAJO FIN DE MÁSTER

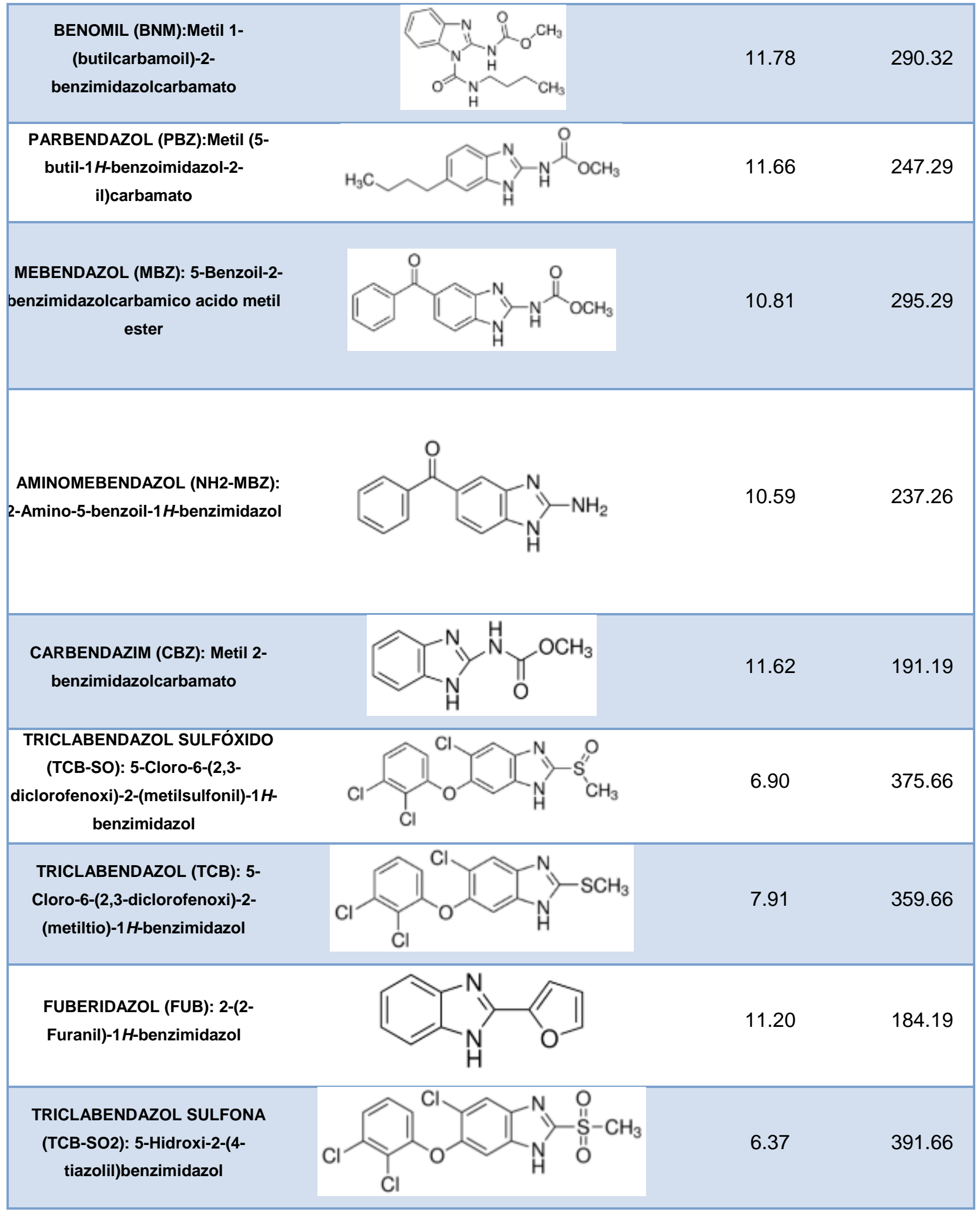




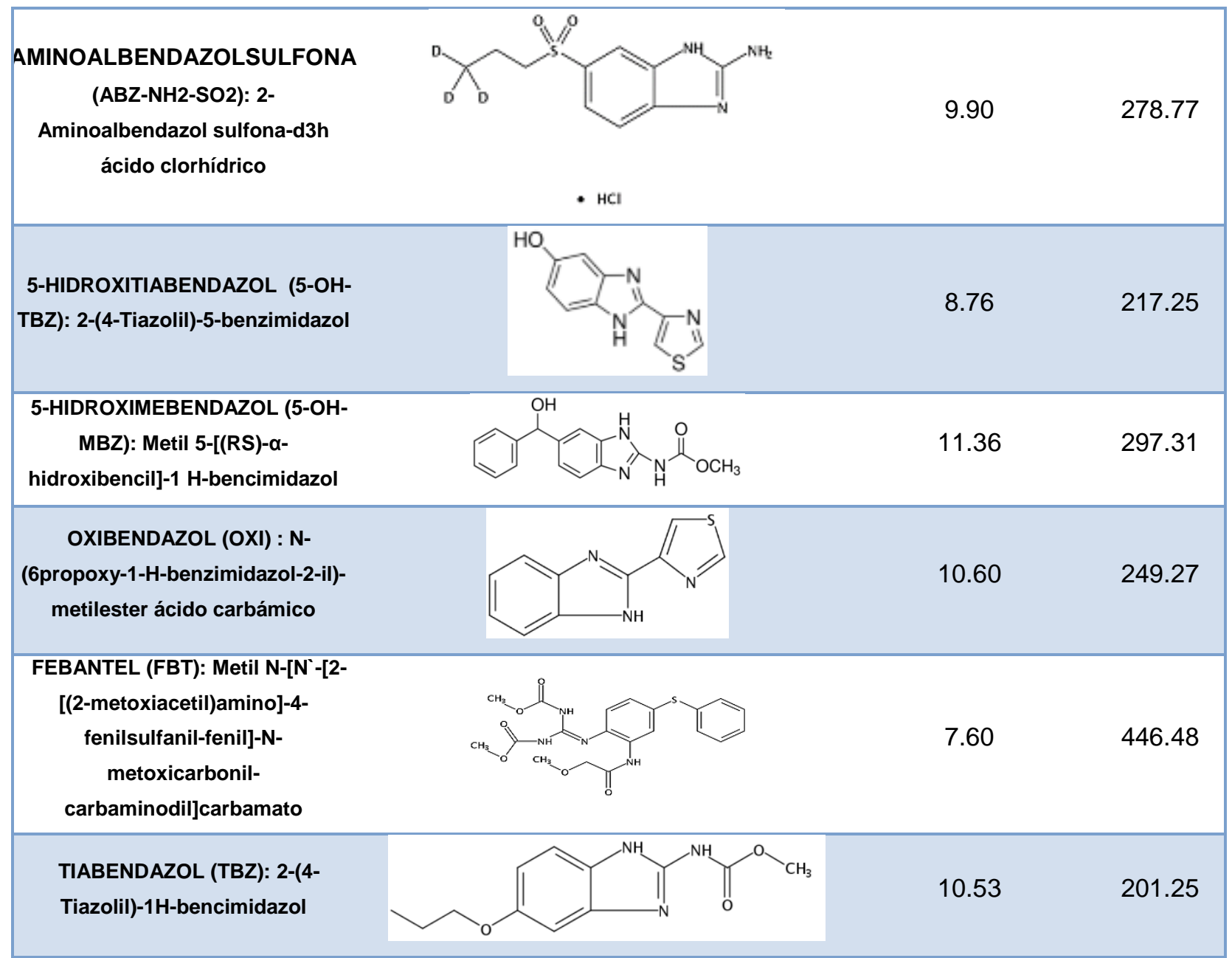

En la tabla se muestran los $\mathrm{p} K_{\mathrm{a}}$ de un número limitado de compuestos cuyos valores aparecen en relación con los átomos de nitrógeno ácido y básico en el anillo de imidazol. Otros benzimidazoles, son moléculas más complejas que poseen más de dos grupos ionizables. MBZ-OH posee grupos hidroxiloácidos. Esto tiene consecuencias importantes para la extracción y limpieza de 5-hidroxitiabendazol (5$\mathrm{OH}-\mathrm{TBZ}$ ) en los residuos de matrices biológicas. Un enfoque común es para llevar a cabo la extracción acuosa de los residuos de baja acidez o, más comúnmente, condiciones alcalinas, antes de ajustar el $\mathrm{pH}$. 


\subsection{LEGISLACIÓN DEL CONTROL DE RESIDUOS DE BENZIMIDAZOLES}

En la actualidad, el control sobre los alimentos y animales es exhaustivo tanto a nivel mundial, como desde la Unión Europea (UE). Es por ello que previo a la investigación y desarrollo del método analítico se ha obtenido información sobre la legislación vigente.

Los documentos oficiales a seguir para conocer y comprobar la legislación vigente son el Libro Blanco sobre Seguridad Alimentaria [12] cuyo objetivo es describir un conjunto de acciones necesarias para completar y modernizar la legislación de la Unión Europea (UE) en el ámbito de la alimentación, con el fin de hacerlo más coherente, más comprensible y más flexible, para garantizar una mejor aplicación de esta legislación y aportar más transparencia a los consumidores. Además garantiza un alto grado de seguridad alimentaria.

Tiene la finalidad de introducir mejoras en los ámbitos del seguimiento y la vigilancia, del sistema de alerta rápida, de la investigación en cuanto a seguridad alimentaria, de la cooperación científica, el apoyo analítico y la formulación de dictámenes científicos, y garantizar una información rápida y fácilmente accesible a los consumidores.

Se deben establecer los LMRs para el control de presencia de contaminantes y residuos a niveles perjudiciales para la salud de los animales y aguas medioambientales. En este caso nos basamos en la legislación comunitaria, donde encontramos la información sobre las normas establecidas en base a la fijación de los LMRs de benzimidazoles (Directiva N. 2377/90/EEC concerniente al establecimiento de LMR para medicamentos de uso veterinario en alimentos de origen animal) [13]. Este documento es un instrumento para documentación y no compromete la responsabilidad de las instituciones. Este documento es un instrumento de documentación y no compromete la responsabilidad de las instituciones.

Se define el LMR como "la máxima concentración de una sustancia química determinada que puede admitirse en un alimento sin que signifique riesgo para la salud" y más específicamente es "la concentración o nivel máximo permitido de un medicamento o sustancia química en un pienso o alimente desde el momento del sacrificio, recolección, procesado, almacenamiento o venta y consumo por animales o el hombre". Una vez que se establecen los LMRs, es necesario establecer en que momento a partir de la aplicación de un producto veterinario, tanto el principio activo como sus metabolitos se hayan eliminado hasta encontrarse en un valor inferior al LMR.

En la tabla siguiente se observa los LMRs en diferentes tejidos del cuerpo en animales:

Tabla 2: LMRs del conjunto de benzimidazoles que vamos a utilizar para el desarrollo del método.

\begin{tabular}{|c|c|c|c|c|}
\hline Benzimidazol & & Especie animal & LMR $(\boldsymbol{\mu g} / \mathbf{k g})$ & Tejidos diana \\
\hline \multirow{2}{*}{ Albendazol } & $\begin{array}{c}\text { Suma de } \\
\text { albendazol } \\
\text { sulfóxido, } \\
\text { albendazol } \\
\text { sulfona y }\end{array}$ & $\begin{array}{c}\text { Todos los } \\
\text { rumiantes }\end{array}$ & 100 & Músculo \\
\cline { 4 - 5 } & & & 100 & Grasa \\
\cline { 3 - 5 } & & & 1000 & Hígado \\
\hline
\end{tabular}


PROPUESTA DE UNA NUEVA METODOLOGÍA PARA EL CONTROL DE RESIDUOS DE BENZIMIDAZOLES MEDIANTE CROMATOGRAFÍA DE LÍQUIDOS CAPILAR

TRABAJO FIN DE MÁSTER

\begin{tabular}{|c|c|c|c|c|}
\hline & $\begin{array}{c}\text { aminoalbendazol } \\
\text { suflfona }\end{array}$ & & 500 & Riñón \\
\hline & & & 100 & Leche \\
\hline \multirow{6}{*}{ Fenbendazol } & & \multirow{6}{*}{$\begin{array}{c}\text { Todas las } \\
\text { especies } \\
\text { destinadas } \\
\text { a la producción } \\
\text { de alimentos, } \\
\text { excepto los } \\
\text { peces }\end{array}$} & 50 & Músculo \\
\hline & & & 50 & Grasa \\
\hline & & & 500 & Hígado \\
\hline & & & 50 & Riñón \\
\hline & & & 10 & Leche \\
\hline & & & 1300 & Huevos \\
\hline \multirow{5}{*}{ Febantel } & \multirow{5}{*}{$\begin{array}{c}\text { Suma de residuos } \\
\text { extraíbles que } \\
\text { por oxidación se } \\
\text { convierten en } \\
\text { sulfona de } \\
\text { oxifebendazol }\end{array}$} & \multirow{4}{*}{$\begin{array}{l}\text { Todos los } \\
\text { rumiantes, } \\
\text { porcinos y } \\
\text { équidos }\end{array}$} & 100 & Músculo \\
\hline & & & 100 & Grasa \\
\hline & & & 1000 & Hígado \\
\hline & & & 500 & Riñón \\
\hline & & $\begin{array}{l}\text { Todos los } \\
\text { rumiantes }\end{array}$ & 10 & Leche \\
\hline \multirow{4}{*}{ Mebendazol } & \multirow{4}{*}{$\begin{array}{l}\text { Adición de } \\
\text { mebendazol }\end{array}$} & \multirow{4}{*}{$\begin{array}{l}\text { Ovinos, caprinos, } \\
\text { équidos }\end{array}$} & 60 & Músculo \\
\hline & & & 60 & Grasa \\
\hline & & & 400 & Hígado \\
\hline & & & 60 & Riñón \\
\hline \multirow{4}{*}{ Oxibendazol } & \multirow{4}{*}{ Oxibendazol } & \multirow{4}{*}{ Porcinos } & 100 & Músculo \\
\hline & & & 500 & Piel y grasa \\
\hline & & & 200 & Hígado \\
\hline & & & 100 & Riñón \\
\hline \multirow{2}{*}{ Tiabendazol } & \multirow{2}{*}{$\begin{array}{l}\text { Suma de } \\
\text { tiabendazol y 5- }\end{array}$} & \multirow{2}{*}{$\begin{array}{r}\text { Bovinos y } \\
\text { caprinos }\end{array}$} & 100 & Músculo \\
\hline & & & 100 & Grasa \\
\hline
\end{tabular}




\begin{tabular}{|c|c|c|c|c|}
\hline \multirow{7}{*}{ Triclabendol } & $\begin{array}{c}\text { hidroxitiabendaz } \\
\text { ol }\end{array}$ & & 100 & Hígado \\
\cline { 4 - 5 } & & & 100 & Riñón \\
\hline & $\begin{array}{c}\text { Conjunto de } \\
\text { residuos } \\
\text { extraíbles que } \\
\text { pueden oxidarse }\end{array}$ & \multirow{2}{*}{$\begin{array}{c}\text { Todos los } \\
\text { rumiantes }\end{array}$} & 225 & Músculo \\
\cline { 4 - 5 } & & 100 & Grasa \\
\cline { 4 - 5 } & & 150 & Hígado \\
\cline { 4 - 5 } & & 10 & Riñón \\
\cline { 4 - 5 } & & 250 & Leche \\
\hline
\end{tabular}

Debido a que nuestro estudio tiene como matriz agua de piscifactoría, debemos hablar del control de residuos de fármacos en el medio ambiente [14]. Los fármacos se han incorporado constantemente en el medio ambiente, o bien por vía animal o mediante residuos orgánicos animales y sin el debido control $[15,16]$ La exposición a fármacos del medio ambiente es función de la combinación de varios factores: cantidad manufacturada, desecho del producto sin usar o una vez caducado, dosis, eficacia de la excreción del compuesto inalterado y de sus metabolitos bioactivos, adsorción/desorción en los suelos y descomposición metabólica una vez sedimentado.

Los antibióticos empleados en piscifactorías se vierten directamente al agua, acumulándose y afectando a los microorganismos acuáticos. El destino de los fármacos en el medio ambiente puede dividirse en tres rutas principales $[17,18]$ :

a) El producto se mineraliza a dióxido de carbono y agua.

b) El producto es lipofílico y parte de él no se degrada, quedando en los sedimentos.

c) El producto se metaboliza a una forma más hidrofílica, pero aún persistente terminando en las aguas residuales o en un río, pudiendo afectar a organismos acuáticos, si los metabolitos son biológicamente activos.

Debido a que durante años son pocos los nuevos fármacos que incluyen un estudio ecotoxicológico apropiado, las nuevas normativas de la UE incluyen el medio ambiente en sus decisiones. En enero de 1998, la UE emitió una guía técnica para controlar el riesgo de la exposición medio ambiental a fármacos empleados en veterinaria (EM/CAMP/055/96-final). Asimismo, la Directiva Técnica 81/852/EEC (enmienda desde el 1 de abril de 1993) concerniente a productos médicos veterinarios, incluye secciones con los requerimientos básicos para hacer un estudio del riesgo de ecotoxicidad medio ambiental, mientras que la guía EMEA/cvmp/055/96-Final de 1997 proporciona las directrices técnicas para la evaluación del riesgo de sustancias médicas veterinarias.

Sin embargo, las directivas técnicas para productos médicos de consumo humano (directiva UE 75/318/EEC) no incluyen ninguna referencia a la ecotoxicidad o ecotoxicología y aparentemente no hay planes de incluirlas próximamente. En este aspecto difiere de su homólogo veterinario (directiva UE 81/852/EEC), descrito anteriormente. 
Así, excepto para los productos que contienen organismos modificados genéticamente, la legislación no especifica cómo realizar una evaluación del riesgo medio ambiental de los productos farmacéuticos.

Es por lo dicho anteriormente que la utilización de fármacos debe de estar bajo un control de residuos tanto en alimentos como en el medio ambiente. Se deben estudiar medidas para eliminar o reducir sus efectos y para ello es necesaria la utilización de técnicas analíticas con elevada sensibilidad y selectividad.

\subsection{OBJETIVO DEL ESTUDIO}

De acuerdo a lo establecido, el objetivo principal de nuestra investigación está enfocado al desarrollo de un método analítico que permita cualificar y cuantificar los benzimidazoles presentes como residuo en animales por debajo de los LMRs. Esta es la razón por la que se quiere llevar a cabo el estudio de benzimidazoles mediante Cromatografía de líquidos capilar.

\subsection{ANTECEDENTES SOBRE LA DETERMINANCIÓN DE BENZIMIDAZOLES}

Los métodos para la determinación de benzimidazoles y sus metabolitos constituyen un trabajo exhaustivo para desarrollar un método analítico multirresiduo. Algunos métodos analíticos encontrados para la determinación de esta familia de compuestos en matrices biológicas y alimentarias son cromatografía de gases, cromatografía en capa fina así como métodos espectrofotométricos directos [19]. Sin embargo, existen inconvenientes en algunas técnicas como la cromatografía de gases ya que los benzimidazoles no son volátiles por lo que se necesita la derivatización de los analitos y por lo tanto requiere más tiempo. Por este inconveniente se prefiere la cromatografía de líquidos. La mayoría de estos métodos emplean columnas de fase inversa $\left(\mathrm{C}_{8}\right.$ ó $\left.\mathrm{C}_{18}\right)$ [19] y modo de elución en gradiente, puesto que si utilizamos modo isocrático no se consigue una buena separación de los analitos [20].

En general, los métodos más empleados utilizan HPLC acoplada a detección por fluorescencia (FL) [21,22], ultravioleta (UV) [23, 24, 25, 26] y espectrometría de masas (MS) [27, 28, 29]. La técnica de electroforesis capilar (CE) acoplada a UV y MS también se ha utilizado para la separación y determinación de benzimidazoles en diversas matrices [30,31, 32, 33]. En el estudio que hemos desarrollado nos basamos en la cromatografía de líquidos capilar para estudiar si la identificación y cuantificación de analitos presenta una mayor ventaja que las técnicas HPLC.

Si hablamos del tratamiento de muestra, la extracción líquida es la más comúnmente utilizada en el análisis de benzimidazoles en distintas matrices de alimentos y matrices biológicas [19]. En los últimos tiempos se ha estudiado la microextracción líquido-líquido dispersiva (DLLME) como alternativa a la extracción líquido-líquido tradicional (LLE) [34]. En este estudio se empleará como tratamiento de muestra la extracción líquido-líquido asistida por sales (SALLE).

\subsubsection{Características de los benzimidazoles}

Los benzimidazoles se administran típicamente como una dosis oral o intraruminal. La biodisponibilidad de benzimidazoles depende en gran medida de la vía de administración y el fármaco en cuestión. Después de la administración de dosis intrarruminales de FBZ y ABZ a ovejas [35], se observa una biodisponibilidad mejorada del fármaco en comparación con dosis orales. En el caso de NBZ, el fármaco se elimina rápidamente del plasma después de $12 \mathrm{~h}$ post-tratamiento, cuando se administra en 
ovejas y ganado vacuno $[36,37,38]$. Este perfil de metabolismo se invirtió después de la administración intrarruminal a la misma dosis.

Las diferencias en la farmacocinética de estos analitos da cuenta de la mayor potencia antihelmíntico. La tasa de distribución depende del peso molecular, solubilidad en lípidos, y la unión a proteínas plasmáticas de cada metabolito de los fármacos. La mayoría de los compuestos de benzimidazol muestran una unión de menos de $50 \%$ a las proteínas plasmáticas, relativamente a alto volumen de distribución, y una tasa de eliminación relativamente rápida.

Debemos de tener en cuenta el metabolismo, el modo de acción, la actividad y la toxicidad de esta familia de compuestos pues el estudio del TFM es un preludio a estudiar su presencia en matrices biológicas.

- Metabolismo de benzimidazoles

Se ha encontrado en estudios con animales que el metabolismo de estos fármacos es extenso, con uno o más metabolitos principales encontrados en el tejido animal o leche para cada compuesto. Los metabolitos que se encuentran dependen de la estructura del fármaco original, la especie de tejido y los animales [39, 40, 41, 42, 43]. Los fármacos ABZ, FBZ, y TCB poseen un enlace de sulfuro, que es susceptible a la oxidación. MBZ posee un grupo carbonilo que puede reducirse para formar un grupo hidroxi. ABZ, FBZ, MBZ poseen grupos carbamato que pueden ser hidrolizados para formar un aminobenzimidazol [44]. TBZ puede oxidarse, dando como resultado la formación de 5-OH-TBZ. Un metabolito 4-hidroxi de TCB y un metabolito ceto también se han observado en la rata, cabra y oveja.

\section{- Modo de acción}

Diferentes modos de acción se han propuesto para los medicamentos de benzimidazol. ABZ se ha demostrado que bloquea la captación de glucosa y disminuye la formación de ATP [45]. El bloqueo de la captación de glucosa ha demostrado ser un mecanismo de acción para MBZ y TBZ [46].

Muchos de los procesos asociados a los benzimidazoles dependen de la integridad de la matriz de microtúbulos [45, 47, 48]. Los microtúbulos están asociados con las siguientes funciones celulares: formación del huso mitótico en la división celular, el mantenimiento de la forma celular, la movilidad celular, la secreción celular, la absorción de nutrientes y el transporte intracelular. En vista de los papeles cruciales, los microtúbulos juegan en muchos procesos celulares su destrucción inducida por fármacos que finalmente conduce a la muerte del organismo. La inhibición de la formación de microtúbulos se ha investigado en detalle con el clásico agente antimitótico colchicina mostrando que este compuesto se une a la tubulina antes de su polimerización [48].

\section{- Actividad}

En las tasas de dosis recomendada en el ganado, TBZ (66 mg/kg de peso corporal) y OXI (10 mg/kg de peso corporal) son eficaces sólo contra los adultos y el desarrollo de fases larvarias de los nematodos. FBZ y ABZ tienen espectros de actividad más amplia que incluyen larvas de adultos. ABZSO y FBZ-SO también son eficaces contra las tenias. TCB es ineficaz contra los nematodos, cestodos. A continuación se muestra una tabla que muestra donde tiene efecto cada benzimidazol en función de la raza animal, es decir la función que tienen como antihelmíntico: 
Tabla 3: Actividades de los bencimidazoles incluyendo el animal tratatado y la diana a tratar.

\begin{tabular}{|c|c|c|}
\hline Benzimidazol & Parásito & Animal \\
\hline$A B Z$ & $\begin{array}{c}\text { Nematodos adultos, tenias } \\
\text { y lombrices } \\
\text { gastrointestinales (Gl) }\end{array}$ & Ovejas \\
\hline \multirow{4}{*}{ FBZ } & $\begin{array}{c}\text { Nematodos pulmonares, } \\
\text { lombrices Gl y tenias }\end{array}$ & Ovejas \\
\hline & $\begin{array}{l}\text { Lombrices GI, gusanos } \\
\text { pulmonares y tenias }\end{array}$ & Cabras \\
\hline & $\begin{array}{l}\text { Lombrices Gl, larva de } \\
\text { nematodos Gl }\end{array}$ & Caballos \\
\hline & Gusanos pulmonares & Cerdos \\
\hline \multirow{2}{*}{ FEB } & $\begin{array}{l}\text { Nematodos pulmonares, } \\
\text { lombrices Gl y tenias }\end{array}$ & Ovejas \\
\hline & $\begin{array}{c}\text { Lombrices Gl y gusanos } \\
\text { pulmonares }\end{array}$ & Cerdos \\
\hline \multirow{3}{*}{ MBZ } & $\begin{array}{c}\text { Gusanos pulmonares y } \\
\text { tenias }\end{array}$ & Ovejas \\
\hline & $\begin{array}{l}\text { Lombrices Gl, gusanos } \\
\text { pulmonares y tenias }\end{array}$ & Cabras \\
\hline & Nematodos y lombrices GI & Aves de corral \\
\hline OXI & Lombriz Gl & Ovejas, caballos, cerdos \\
\hline
\end{tabular}




\begin{tabular}{|ccc|}
\hline & $\begin{array}{c}\text { Gusanos pulmonares, } \\
\text { lombrices GI }\end{array}$ & Ovejas \\
\cline { 2 - 3 } & Lombrices GI, P. equorum & Caballos \\
\hline TCB & Trematodos & Ovejas \\
\hline
\end{tabular}

- Toxicidad

Los benzimidazoles son considerados como seguros hasta de 20 a 30 veces la dosis recomendada. La toxicidad aguda es difícil de inducir con estos. Los informes de toxicidad aguda de benzimidazoles en los animales son muy limitados [50]. El principal efecto tóxico de los compuestos de benzimidazol implica su efecto teratogénico. Malformaciones congénitas que resultan de la administración de antihelmínticos de benzimidazol durante la gestación en ovejas se han observado con FBZ [51] y ABZ [52]. Sin embargo, FBT [53], FBZ [54], MBZ [55] y OXI [56, 57] no parecen ejercer un efecto teratogénico en ovejas cuando se administra en la gestación temprana. En general, si estos fármacos se van a utilizar en el embarazo temprano, tiene que ser por una buena razón y en las dosis más baja recomendada. De particular interés es el hecho de que algunos metabolitos de benzimidazol son más tóxicos que el fármaco original; por ejemplo, hidroxi-mebendazol (OH-MBZ) se ha encontrado para ser más embriotóxico que MBZ en la rata [58].

- Propiedades físicas y químicas importantes de benzimidazoles

Los benzimidazoles son la familia química más grande utilizada para tratar enfermedades endoparásitarias en animales domésticos. Este grupo incluye análogos de tiabendazol y carbamatos de benzimidazol. Mucha información sobre las propiedades físicas y químicas de los benzimidazoles se puede encontrar en los libros de referencia, a partir de proveedores de productos químicos y mediante cálculos sencillos. Los valores $\mathrm{p} K_{\mathrm{a}}$ en particular, proporcionan información importante sobre las propiedades de solubilidad [59, 60-65]. Se sabe que benzimidazoles poseen un anillo de imidazol que contiene ambos átomos de nitrógeno ácido y básico. En condiciones adecuadas, la molécula puede estar protonado ( $p K_{\text {un }} \sim 5-6$ ) o desprotonado ( $p K_{\text {un }} \sim 12$ ) [64], como se muestra en la siguiente figura:

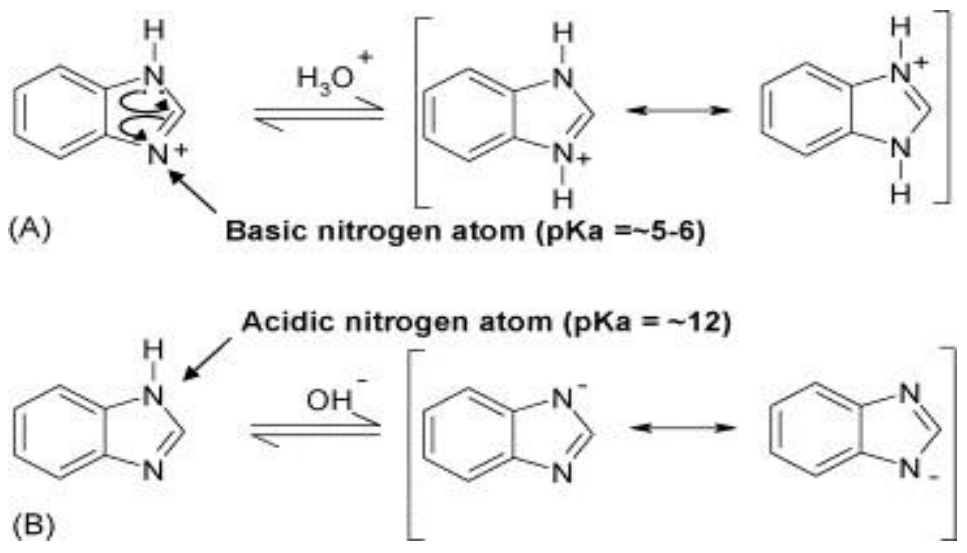

Figura 1: La ionización de la molécula de ácido benzimidazol bajo (A) y las condiciones básicas (B) [19] 


\section{PROPUESTA DE UNA NUEVA METODOLOGÍA PARA EL CONTROL DE RESIDUOS DE BENZIMIDAZOLES \\ MEDIANTE CROMATOGRAFÍA DE LÍQUIDOS CAPILAR}

\section{TRABAJO FIN DE MÁSTER}

\subsubsection{Cromatografía de líquidos capilar}

La cromatografía de líquidos capilar (CLC) como otras técnicas de separación miniaturizadas está en el foco de los métodos analíticos modernos y, en particular, en el ámbito de bioanálisis y ciencias de la vida. Las ventajas más importantes de estos sistemas en comparación con técnicas clásicas son la reducción del consumo de disolventes, el impacto ambiental reducido, los pequeños volúmenes de muestra y el acoplamiento con la detección MS. Dentro de estas técnicas, un punto crucial es la elección de la columna capilar que se utiliza con respecto a la estabilidad de la columna y vida, así como la facilidad de fabricación. Con esta técnica se han obtenido buenos resultados para la determinación de micotoxinas en vino [65, 66, 67] y cereal [68] Aplicaciones de la cromatografía líquida capilar desarrolladas en nuestro grupo de investigación: análisis de micotoxinas en vino [76]. En el control de residuos de antibióticos en alimentos de origen animal [77] En la determinación de residuos de plaguicidas en aguas, frutas y vegetales [78-82].

Como se puede observar en la Tabla 4 la CLC permite utilizar columnas de diámetro reducido y permite flujos de fase móvil muy bajos por lo que el consume de disolventes es mínimo. En el método analítico propuesto, se ha aplicado la CLC en fase inversa mediante elución en gradiente.

\begin{tabular}{|c|c|c|c|c|}
\hline Parámetro & HPLC & Micro LC & LC Capilar & Nano LC \\
\hline $\begin{array}{c}\text { Diámetro interno de las } \\
\text { columnas }\end{array}$ & $1.5-4.6 \mathrm{~mm}$ & $0.5-1.5 \mathrm{~mm}$ & $0.18-0.32 \mathrm{~mm}$ & $0.010-0.150 \mathrm{~mm}$ \\
\hline $\begin{array}{c}\text { Longitud del lecho } \\
\text { cromatográfico }\end{array}$ & $3-30 \mathrm{~cm}$ & $5-20 \mathrm{~cm}$ & $5-15 \mathrm{~cm}$ & $15-15 \mathrm{~cm}$ \\
\hline $\begin{array}{c}\text { Diámetro medio de } \\
\text { partículas }\end{array}$ & $3-10 \mu \mathrm{m}$ & $3-5 \square \mathrm{m}$ & $3-5 \mu \mathrm{m}$ & $5 \mu \mathrm{m}$ \\
\hline Flujo de fase móvil & $0.2-2.5 \mathrm{ml} / \mathrm{min}$ & $10-100 \mu \mathrm{l} / \mathrm{min}$ & $1-10 \mu \mathrm{l} / \mathrm{min}$ & $0.1-1 \mu \mathrm{l} / \mathrm{min}$ \\
\hline
\end{tabular}

Tabla 4: Características de las distintas técnicas de cromatografía líquida.

\subsubsection{Tratamiento de muestra}

A continuación explicamos los dos tratamientos de muestra que hemos evaluados en este TFM.

- Microextracción líquido-líquido dispersiva (DLLME)

La microextracción líquido-líquido dispersiva se trata de un método de tratamiento de muestra novedoso utilizado para la pre-concentración de muestra [69]. Se trata de una técnica emergente introducida para el tratamiento de muestras líquidas [70,71, 72, 73]. Esta técnica se basa en el uso de un sistema con tres disolventes, en donde una mezcla apropiada de unos pocos microlitros permite la extracción de los analitos. Este sistema se compone de un disolvente de extracción orgánico (por lo general con una densidad mayor que el agua), y un pequeño volumen de un disolvente dispersor (miscible con el disolvente de extracción y con agua), esta disolución se inyecta rápidamente en una muestra acuosa, lo que resulta una formación de una microemulsión donde los analitos a estudiar se extraen rápidamente en el disolvente de extracción. La separación de fases se realiza por centrifugación y en la fase orgánica es donde encuentran los analitos de interés que se encuentra en el fondo de un tubo falcon.

Presenta alto rendimiento, y es una técnica rápida y barata. Desde un punto de vista comercial, económico y ambiental, las ventajas de DLLME respecto a los métodos de extracción con disolventes 
convencionales son la simplicidad de operación, rapidez, bajo costo, fácil manipulación, pequeñas cantidades de disolventes de extracción orgánicos, altas recuperaciones y los factores de enriquecimiento, y la vinculación más fácil para las técnicas. Es una buena técnica para desarrollar procedimientos sencillos y rápidos que proporcionan suficiente sensibilidad para detectar cantidades muy bajas de analitos en muestras biológicas.

- Extracción líquido-líquido asistida con sales (SALLE)

La extracción liquido-líquido (SALLE) proporciona una alternativa viable para la preparación de muestras con el objetivo de obtener una pre-concentración de muestra sin gastan grandes cantidades de disolventes orgánicos o emplear demasiado tiempo en el tratamiento de muestra. Los analitos que son objeto del estudio se extraen de la fase orgánica. Mediante este tratamiento de muestra basado en el tratamiento LLE, una concentración adecuada de sal se añade para permitir la separación entre la fase acuosa y fase orgánica, siendo en esta última parcialmente miscible [75].

Algunos disolventes utilizados son acetonitrilo, acetona, acetato de etilo, etc. Además su uso está proyectado para la preparación de muestras de agua con alta salinidad. Es un método de tratamiento de muestra fácil, con un consumo mínimo de reactivos y no muy caros. Además el tiempo de preparación es mínimo, siendo la eliminación de $\mathrm{MeCN}$ mediante secado por corriente de $\mathrm{N}_{2}$ lo que más tiempo conlleva [74].

\section{OBJETIVOS ESPECÍFICOS}

Los objetivos específicos del Trabajo Fin de Máster se pueden enumerar en función de los conocimientos que se necesitan para lograr el objetivo final.

- Conocer las características y propiedades físico-químicas de la familia de compuestos benzimidazoles.

- Conocer la legislación vigente dentro del marco europeo para saber los LMRs de dichos compuestos para alimentos de origen animal e igualmente conocer la problemática que conlleva la presencia de residuos de fármacos de uso veterinario en el medioambiente, y para los que no existe LMRs.

- Buscar información de la técnica empleada, siendo en nuestro caso cromatografía líquida capilar, tanto de los fundamentos de la misma como del equipo empleado.

- Familiarizarnos con el software a través del cual ejecutamos las acciones en el equipo y más tarde analizamos los resultados de cromatogramas.

- El objetivo principal, siendo la optimización del método analítico, lleva consigo unas variables que se optimizan secuencialmente: longitud de onda de estudio de cromatograma fase móvilgradiente-flujo-temperatura de columna.

\section{METODOLOGÍA: MATERIAL Y MÉTODOS}

\subsection{TIPO DE DISEÑO Y ESTUDIO}

El tipo de estudio y diseño se considera en base al objetivo principal del trabajo, que consiste en la separación de un número de analitos pertenecientes a la familia de los benzimidazoles. Es por ello que 
se trabaja con un método de separación como la cromatografía de líquidos. A su vez, dado que el estudio de benzimidazoles se ha realizado en trabajos anteriores con otras técnicas cromatográficas, se selecciona la cromatografía de líquidos capilar. Cuando estos compuestos se encuentran como residuos en aguas medioambientales su concentración es muy baja y por ello se necesitan tratamientos de muestra eficaces capaces de alcanzar factores de pre-concentración elevados.

\subsection{RECURSOS}

Los recursos a los que hemos tenido accesos se basan en un equipo de cromatografía de líquidos capilar que se encontraba en el laboratorio de instrumentación y del material necesario para la preparación de disoluciones y preparación de muestra en un laboratorio contiguo. Dentro del laboratorio de tratamiento de muestras se ha facilitado el material necesario para llevar a cabo la preparación de disoluciones y muestras necesarias para abarcar el método analítico.

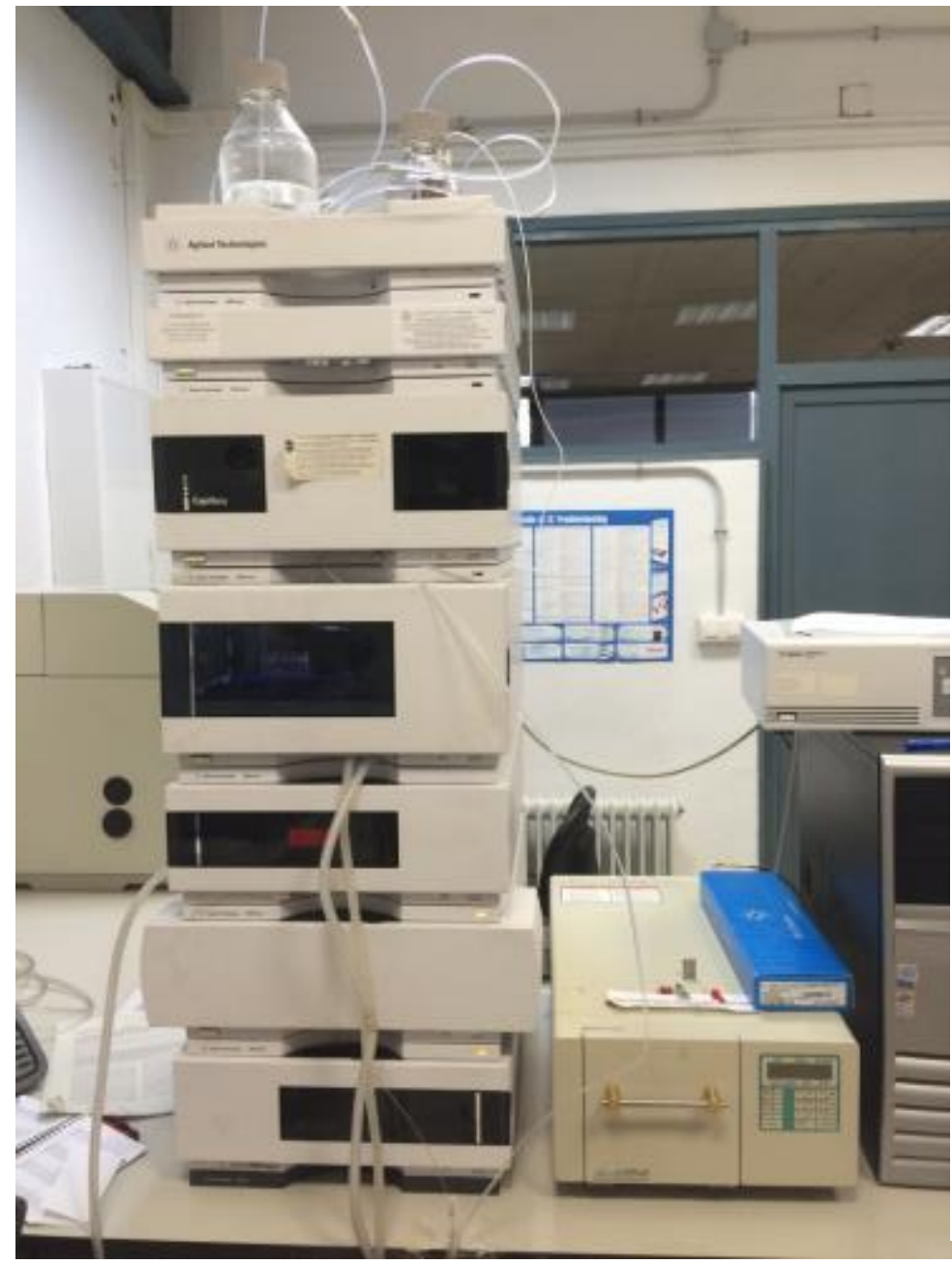

El equipo utilizado para llevar a cabo el análisis experimental se trata de un sistema de cromatografía de líquidos capilar, marca Agilent Technologies, modelo 1200. Equipado con una bomba capilar con sensor de flujo capilar (máximo caudal: $20 \mu \mathrm{L} / \mathrm{min})$, automuestrador con una placa del inyector automático micro pocillos con un dispositivo de microdosificación que proporciona

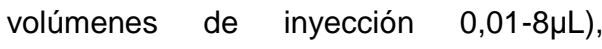
contiene un desgasificador automáticos (parte G1379B Agilent), junto con horno de columnas y detector de bacteria de diosdos (DAD). La columna que se utiliza es Zorbax XDB-C18 (Agilent Technologies) de fase inversa $(150 \mathrm{~mm}$. $0.5 \mathrm{~mm}, 5 \mu \mathrm{m}$ tamaño de partícula). La toma de datos se lleva a cabo mediante el programa Agilent ChemStation (Rev. A.10.20, Agilent Technologies, Alemania).

Fotografía 1: Equipo de Cromatografía de líquidos capilar del Laboratorio del Grupo de Investigación FQM 302 de la Universidad de Granada. 
En cuanto a la preparación de las distintas disoluciones tenemos que tener en cuenta:

- Para la preparación de la fase móvil se utilizan reactivos de grado analítico, entre los que están el acetonitrilo (MeCN), metanol (MeOH) adquiridos en VWR Chemicals-Prolabo BDH; el acetato amónico. Además estos disolventes también se utilizaron para la preparación de disoluciones que se iban a inyectar.

- El agua empleada ha sido agua ultrapura $\left(18.2 \mathrm{M}^{\prime} \Omega \mathrm{cm}^{-1}\right)$ que se obtiene en un sistema Mili-Q (Millipore, Bedford,MA,EEUU).

- Para la preparación de la disolución utilizada para optimizar el método, se partió de una disolución de 10ppm que contenía los 21 analitos en estudio y se preparó una de trabajo de concentración $2 \mathrm{ppm}$ en cada analito. Con esta concentración se realizaron las distintas pruebas para optimizar los parámetros del método.

- La identificación de los 21 benzimidazoles se realizó a partir de disoluciones madre de cada uno, preparadas en metanol, a las concentraciones que se muestran a continuación. En la tabla se indica el volumen de cada uno de ellos para conseguir una concentración final de 20ppm en la disolución de trabajo con un volumen final de $2 \mathrm{~mL}$. A partir de esta disolución se preparó una disolución de $5 \mathrm{ppm}$ diluyendo la anterior con los volúmenes necesarios. En este paso se prepararon 21 viales, todos con la misma concentración. Los volúmenes necesarios de cada uno de ellos se muestran:

\begin{tabular}{|ccc|}
\hline ANALITO & $\begin{array}{l}\text { Concentración de las } \\
\text { disoluciones madre } \\
(p \text { pm) }\end{array}$ & Volumen $(\mu L)$ \\
\hline $5-O H-T B Z$ & 1000 & 40 \\
\hline NH2-ABZ-SO2 & 1000 & 40 \\
\hline ABZ-SO & 1000 & 40 \\
\hline BNM+CBZ & 500 & 80 \\
\hline TBZ & 1000 & 40 \\
\hline FUB & 500 & 40 \\
\hline FBZ-SO & 1000 & 40 \\
\hline NH2-MBZ & 1000 & 40 \\
\hline $5-O H \_M B Z$ & 500 & 80 \\
\hline FBZ-SO2 & 250 & 160 \\
\hline
\end{tabular}


PROPUESTA DE UNA NUEVA METODOLOGÍA PARA EL CONTROL DE RESIDUOS DE BENZIMIDAZOLES

MEDIANTE CROMATOGRAFÍA DE LÍQUIDOS CAPILAR

TRABAJO FIN DE MÁSTER

\begin{tabular}{|ccc|}
\hline MBZ & 250 & 160 \\
\hline ABZ & 500 & 80 \\
\hline TCB-SO2 & 1000 & 40 \\
\hline FBZ & 250 & 160 \\
\hline TCB-SO & 1000 & 40 \\
\hline TCB & 1000 & 40 \\
\hline
\end{tabular}

Tabla 5: Concentración de las disoluciones madre de cada analito preparadas en metanol (columna 1) y volumen para la preparación de la disolución madre de $5 \mathrm{ppm}$ con volume final de $2 \mathrm{~mL}$ (columna 2 ).

Una vez que se han identificado los benzimidazoles, se lleva a cabo una selección de 17 de ellos debido al solapamiento de picos de algunos y para la selección seguimos unas premisas que más tarde explicaremos. Los parámetros que quedan por optimizar se realizan con estos 17 analitos. Para ello se prepara una disolución de trabajo que contengan a los 17 benzimidazoles elegidos para una concentración final de $5 \mathrm{ppm}$ en $2 \mathrm{~mL}$. Para preparar esta concentración, se toma un volumen de cada analito, conociendo las concentraciones en las que están preparados, mostrado anteriormente.

- Para la preparación de la recta de calibrado de patrones, se preparan cinco disoluciones que contienen los 17 analitos a cinco concentraciones y cuyo disolvente está compuesto por $70 \%$ agua y $30 \%$ fase orgánica (MeOH:MeCN, 50:50). Los volúmenes son los siguientes:

\begin{tabular}{|ccc|}
$\begin{array}{c}\text { Concentración } \\
(\mathrm{ppb})\end{array}$ & Volumen agua( $\mu \mathrm{L})$ & $\begin{array}{c}\text { Volumen fase } \\
\text { orgánica( } \mu \mathrm{L})\end{array}$ \\
\hline $\mathbf{1 0}$ & 686 & 294 \\
\hline $\mathbf{2 5 0}$ & 665 & 285 \\
\hline $\mathbf{5 0 0}$ & 630 & 270 \\
\hline $\mathbf{7 5 0}$ & 595 & 255 \\
\hline 1000 & 500 & 240 \\
\hline
\end{tabular}

Tabla 6: Muestra las cantidades de disolución de analitos y disolvente (agua y fase orgánica) para la preparación de los patrones para la realización de la recta de calibrado

Los viales que preparamos de todas las disoluciones inyectadas en el equipo tienen un volumen final de $1 \mathrm{~mL}$. 
TRABAJO FIN DE MÁSTER

\section{- PREPARACIÓN DE MUESTRAS}

Como ya hemos explicado en los antecedentes, el tratamiento de muestra se prueba con dos métodos, los pasos a seguir para cada uno de los dos se muestran a continuación:

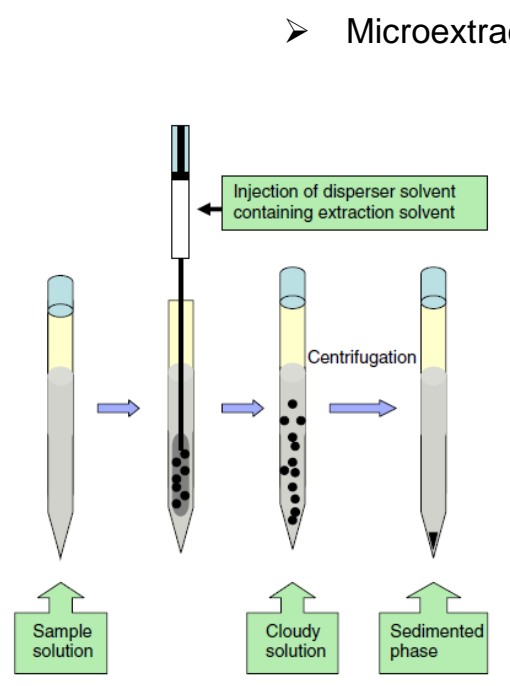

El procedimiento a seguir es: en un tubo falcon de $15 \mathrm{~mL}$ se añaden $5 \mathrm{~mL}$ de muestra (agua) más $100 \mu \mathrm{L}$ de la disolución de benzimidazoles (5ppm) y se lleva a vortex durante $2 \mathrm{~min}$. En un tubo de $5 \mathrm{~mL}$ se introducen $1700 \mu \mathrm{L}$ de $\mathrm{MeOH}$ (dispersante) y $950 \mu \mathrm{L}$ de cloroformo (extractante). La mezcla de ambos se inyecta en el tubo falcon produciendo la microemulsión. A continuación se lleva a la centrifuga (6000rpm, 5min) para la separación de fases. Con jeringa se extrae la fase orgánica que se encuentra en el fondo al ser más densa que el agua y se introduce en un tubo de cristal de $5 \mathrm{~mL}$ para el secado (Corriente de $\mathrm{N}_{2}$ ). Una vez que se ha evaporado todo se añade $300 \mu \mathrm{L}$ mezcla $B$ fase móvil. Tras 1 min en vortex se añade $700 \mu \mathrm{L}$ de agua y otra vez vortex $2 \mathrm{~min}$. Por último se lleva al vial de análisis, filtrándose previamente la disolución. Este vial se mantiene en el vortex 2 min.

Extracción líquido-líquido asistida por sales (SALLE):

En este tratamiento el procedimiento es más simple: en un tubo falcon de $15 \mathrm{~mL}$ añadimos $4 \mathrm{~mL}$ de muestra (agua) más $266 \mu \mathrm{L}$ de disolución de benzimidazoles $5 \mathrm{ppm}$ y a continuación $8 \mathrm{~mL}$ MeCN. Además adicionamos $1.6 \mathrm{~g}$ de $\mathrm{NaCl}$. Esta disolución se lleva al vortex hasta que la sal se disuelva. Luego se centrifuga (7500rpm, $10 \mathrm{~min}$ a $25^{\circ} \mathrm{C}$ ). Tras la separación de fases, con jeringa cogemos $3 \mathrm{~mL}$ de la fase superior y se lleva a secado. Una vez que el MeCN se ha eliminado se reconstituye con 70:30 de agua:faseorgánica (MeOH:MeCN, 50:50). A continuación se filtra y el vial se mantiene en vortex durante $2 \min$.

\subsection{FASES DEL ESTUDIO}

Dado que el TFM se ha basado en la optimización de un método analítico junto con la aplicación de dicho método para la determinación de residuos de benzimidazoles en agua medioambientales, las fases de estudio que hemos seguido son:

> Optimización de parámetros del método, incluyendo flujo de la fase móvil, temperatura de la columna y volumen de inyección de la miestra, manteniendo la naturaleza de la fase móvil y el gradiente que había sido establecidos en un estudio anterior en el grupo.

$>$ Establecimiento de la recta de calibrado con patrones de los benzimidazoles seleccionados.

> Evaluación de tratamientos de muestras, comparando la microextracción líquido-líquido dispersiva y la extracción líquido-líquido asistida por sales. El estudio tendrá lugar en una muestra de agua MiliQ y en agua de piscifactoría.

> Validación del método mediante el establecimiento de los parámetros de calidad y la realización de un calibrado en presencia de matriz (con muestras de agua de piscifactoría). 


\section{RESULTADOS Y DISCUSIÓN}

Los resultados que se presentan a continuación parten de un estudio previo realizado en el grupo de investigación FQM 302 en el que se pretendía poner de manifiesto el potencial de la CLC para la determinación de benzimidazoles mediante CLC. En este TFM se ha partido del estudio inicial siendo el objetivo del trabajo experimental la puesta a punto de un nuevo método analítico para la determinación de residuos de benzimidazoles en aguas medioambientales. La técnica de CLC se emplea acoplada a un detector UV de batería de diodos (DAD), puesto que estos compuestos tienen la capacidad de absorber radiación en esta región. Partiendo de unas condiciones iniciales, llevamos a cabo la optimización de diferentes parámetros.

\section{$\rightarrow$ OPTIMIZACIÓN DE LA SEPARACIÓN CROMATOGRÁFICA:}

Inicialmente se lleva a cabo el estudio de la fase móvil y el gradiente, partiendo de las condiciones iniciales establecidas en el grupo de investigación en el que se ha llevado a cabo el trabajo. Las características de la fase móvil se van modificando con objeto de conseguir la separación eficar de los analitos.

\subsection{CONDICIONES INICIALES}

Las condiciones iniciales de las que partimos son:

\begin{tabular}{cc}
\hline Volumen inyección & $8 \mu \mathrm{L}$ \\
\hline Temperatura columna & $20^{\circ} \mathrm{C}$ \\
Longitud de onda & $290 \mathrm{~nm}$ \\
Flujo fase móvil & $7 \mu \mathrm{L} / \mathrm{min}$ \\
Fase móvil & $70 \%$ F.acuosa:Tampón acetato amónico 50nM \\
& $30 \%$ F. Orgánica: MeOH:MeCN(50:50) \\
\hline Gradiente & \\
\hline
\end{tabular}

Tiempo (minutos)

0.00

14.00

17.00

19.00

29.00

39.00

42.00
F. Orgánica (\%)

30.0

40.0

70.0

95.0

95.0

30.0

30.0

Tabla 7: Condiciones iniciales a partir de las que optimizamos el método.

\subsubsection{Gradiente}

Tras un estudio de diferentes gradientes, donde se comienza con un porcentaje de fase móvil del $5 \%$ hasta llegar al $40 \%$, manteniendo los tiempos de análisis como se observa en la tabla inferior, se observa que al aumentar el porcentaje de disolvente orgánico en la fase móvil en el inicio del gradiente se produce una salida de los analitos de la columna con mayor rapidez. Sin embargo, si aumentamos el porcentaje hasta $40 \%$, este $10 \%$ superior a lo establecido finalmente provoca que parte de los analitos más polares, y que tiene una menor interacción con la fase estacionaria salgan solapados. Por otro lado, 
para conseguir que todos los analitos eluyan antes de la etapa de lavado de la columna debemos de mantener un intervalo de tiempo al $95 \%$ de fase orgánica. En este caso, se estableció un periodo de $10 \mathrm{~min}$.

Más tarde, dentro de nuestro propio estudio se comprobó que para estar de acuerdo a lo decido el flujo debe ser mayor, sino parte de los analitos salen a tiempos donde ya se vuelve a aplicar el $30 \%$ de fase orgánica, y por tanto estamos en fase de lavado. Las características de realizar una elución en gradiente se muestran en la tabla siguiente:

\begin{tabular}{cc}
\hline Tiempo (minutos) & F. Orgánica (\%) \\
\hline 0.00 & 30.0 \\
14.00 & 40.0 \\
17.00 & 70.0 \\
19.00 & 95.0 \\
29.00 & 95.0 \\
39.00 & 30.0 \\
42.00 & 30.0 \\
\hline
\end{tabular}

Tabla 8: Características en tiempo y \% de fase orgánica aplicadas a la elución en gradiente.

\subsubsection{Fase móvil}

Dentro del estudio de la fase móvil, debemos de tener en cuenta la fase orgánica y la fase acuosa. Lo establecido en las condiciones iniciales son:

30\% Fase orgánica compuesta por metanol (MeOH) y acetonitrilo (MeCN) en proporciones 50:50 70\% Fase acuosa compuesta por tampón de acetato Amónico $50 \mathrm{mM}$

- Fase orgánica

En estudios previos se realizaron pruebas con fases orgánicas compuestas por MeCN, metanol y etanol. Los cromatogramas obtenidos presentaban inconvenientes, aquellos que permitían la identificación de más analitos no presentaban resoluciones en la mayoría superiores a 1.5; y si se identificaban menos analitos la resolución aumentaba. Por ello se optó por condiciones intermedias a las estudiadas, teniendo una fase móvil final compuesta por $\mathrm{MeCN}: \mathrm{MeOH}$ al 50:50. En estas condiciones se obtenían 17 analitos, aunque en el caso de 3 con una resolución menor a 1.5.

- Fase acuosa

La fase acuosa está compuesta por un Tampón de Acetato Amónico 50mM. Debido a que la variación del $\mathrm{pH}$ tiene influencia en la separación de los bencimidazoles se utiliza esta disolución como tal. Se comprobó con disoluciones acuosas con un $0.1 \%$ de ácido fórmico y se comprobó un solapamiento de aquellos picos correspondientes a los benzimidazoles más polares. Esto es debido a que pH ácido, los analitos con carga positiva son menos retenidos y por tanto eluyen antes de forma conjunta.

\subsection{PARÁMETROS A OPTIMIZAR}

\subsubsection{Características de la disolución}

En nuestro estudio, comenzamos preparando un vial con una concentración de 2ppm de los 21 analitos inicialmente considerados. 


\section{TRABAJO FIN DE MÁSTER}

Comenzamos nuestro estudio con las condiciones descritas anteriormente, comprobando la reproducibilidad de las mismas durante varias inyecciones. Cada una de las inyecciones se repite dos veces para comprobar la reproducibilidad de los resultados.

Tras comprobar que los resultados son reproducibles, comenzamos con la optimización de las variables a considerar.

- Cromatograma en las condiciones iniciales a una concentración de 2 ppm:

Cromatograma en obtenido en les condiciones iniciales

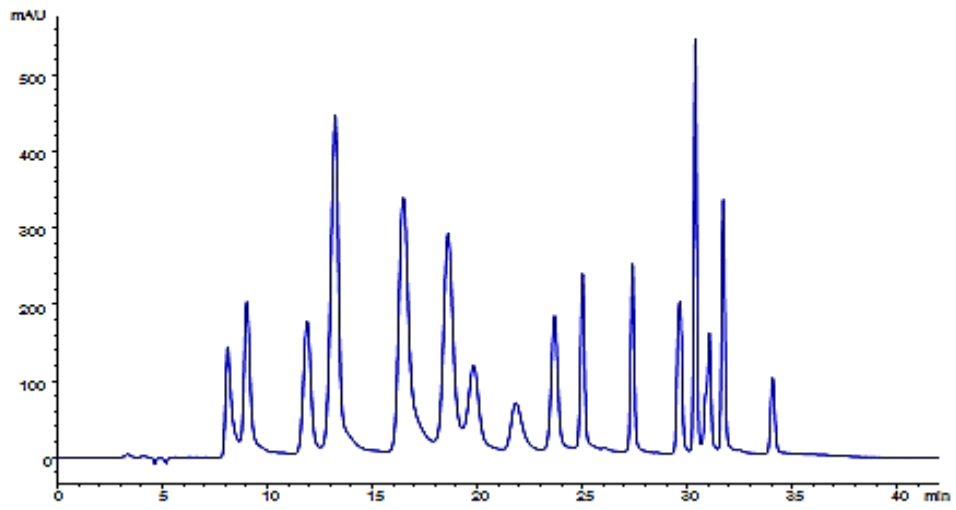

Figura 2: Cromatograma en condiciones iniciales en una disolución de trabajo con los 21 anaitos en estudio.

Antes de seguir con el estudio se inyecta un blanco con metanol únicamente para saber las interferencias que podemos tener en las condiciones empleadas.

Cromatograma realizado para el blancocon una disolución de metanol

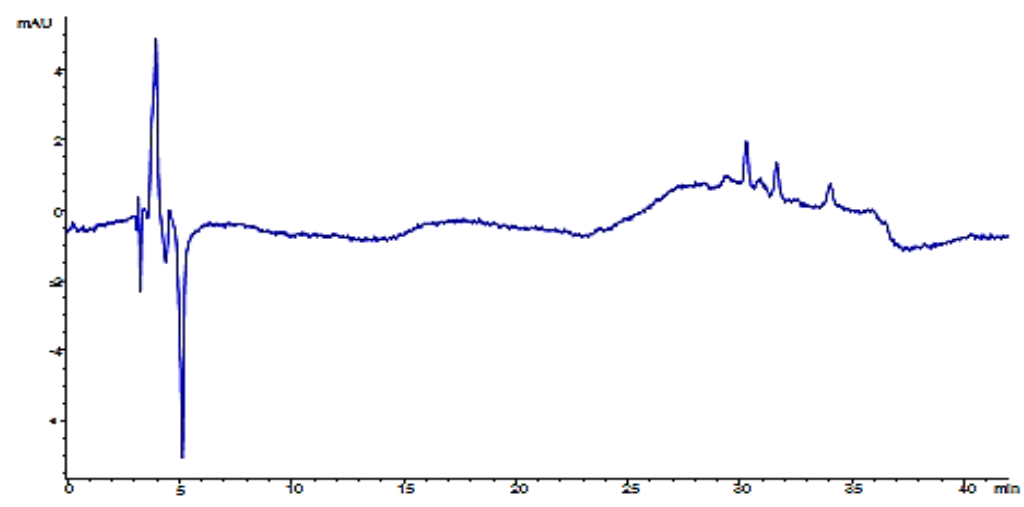

Figura 3: Cromatograma del blanco de disolvente. 


\section{TRABAJO FIN DE MÁSTER}

\subsubsection{Flujo de la fase móvil}

Se estudió el flujo de la fase móvil, haciendo un barrido desde $7 \mu \mathrm{L} / \mathrm{min}$ a $17 \mu \mathrm{L} / \mathrm{min}$ aumentando en $2 \mu \mathrm{L} /$ min cada inyección. Este estudio se llevó a cabo con objeto de intentar mejorar la resolución de algunos picos. Sin embargo, el aumento del flujo lleva consigo un solapamiento de picos, junto con un empeoramiento de resolución de los mismos en algunos casos.

Es por ello, que tras realizar el aumento de flujo, y antes de optimizar cualquier parámetro se llevó a cabo la identificación de los analitos. Para la identificación de los analitos, se prepararon viales con cada analito por separado a un volumen final de $1 \mathrm{~mL}$ y concentración de $2 \mathrm{ppm}$.

A continuación se muestras los distintos cromatogramas a diferentes flujos, indicando los analitos presentes:

$>$ IDENTIFICACIÓN DE ANALITOS

Los resultados obtenedos se muestran en los cromatogramas siguientes:

- Flujo $7 \mu \mathrm{L} / \mathrm{min}$

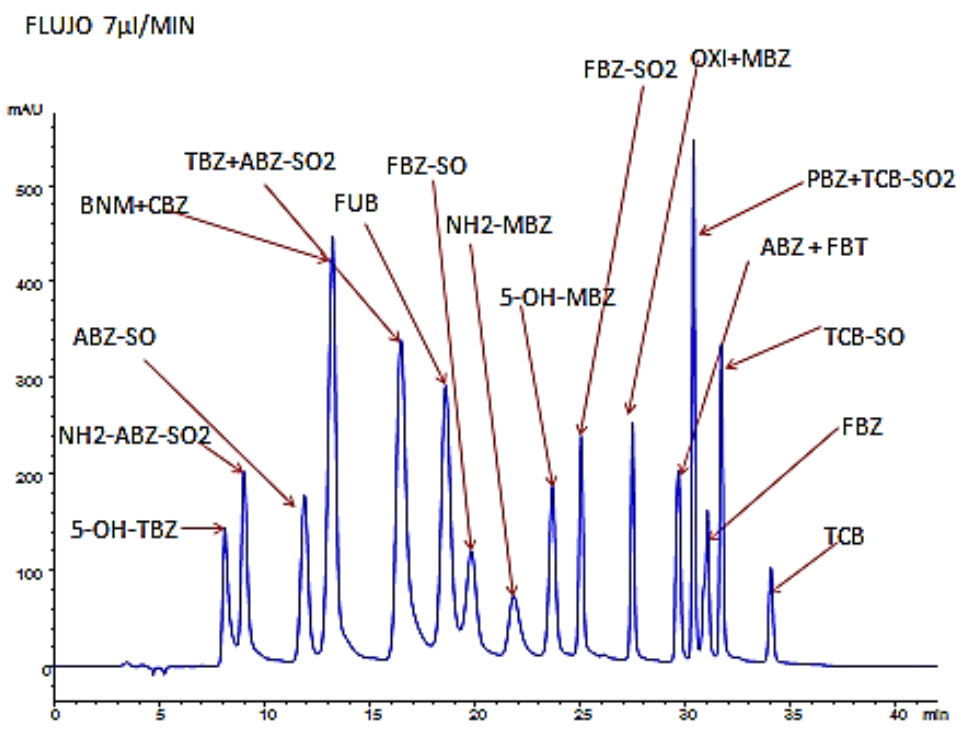

Figura 4: Cromatogramas para la optimización de la velocidad de flujo. 
- Flujo $9 \mu \mathrm{L} / \mathrm{min}$

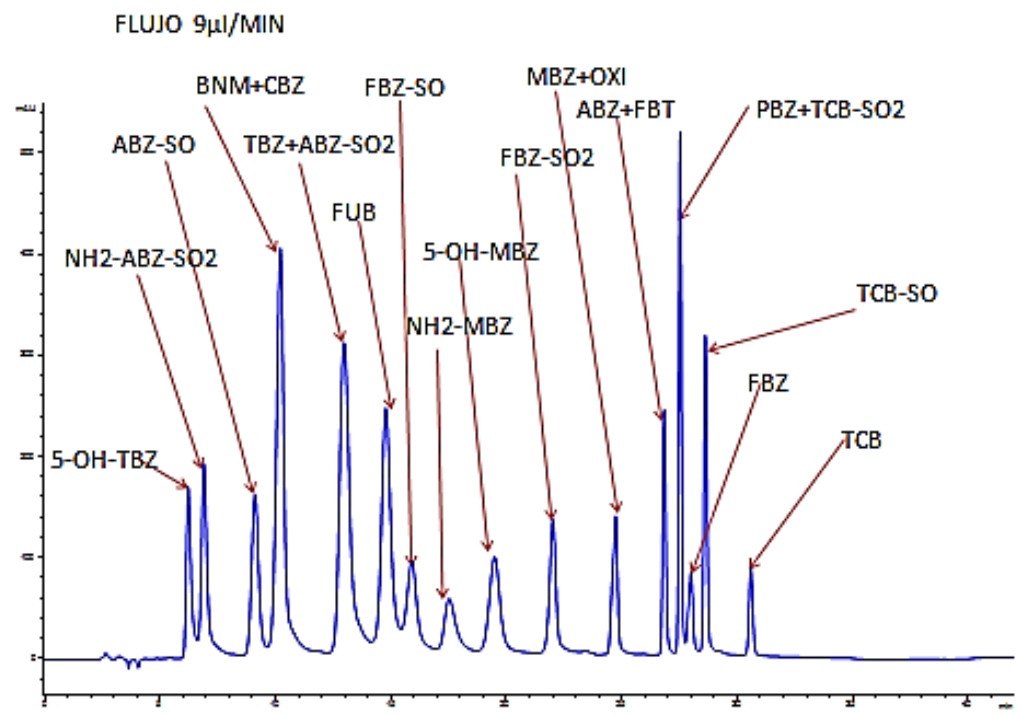

- Flujo $11 \mu \mathrm{L} / \mathrm{min}$

FLUJO $11 \mu \mathrm{l} / \mathrm{MIN}$

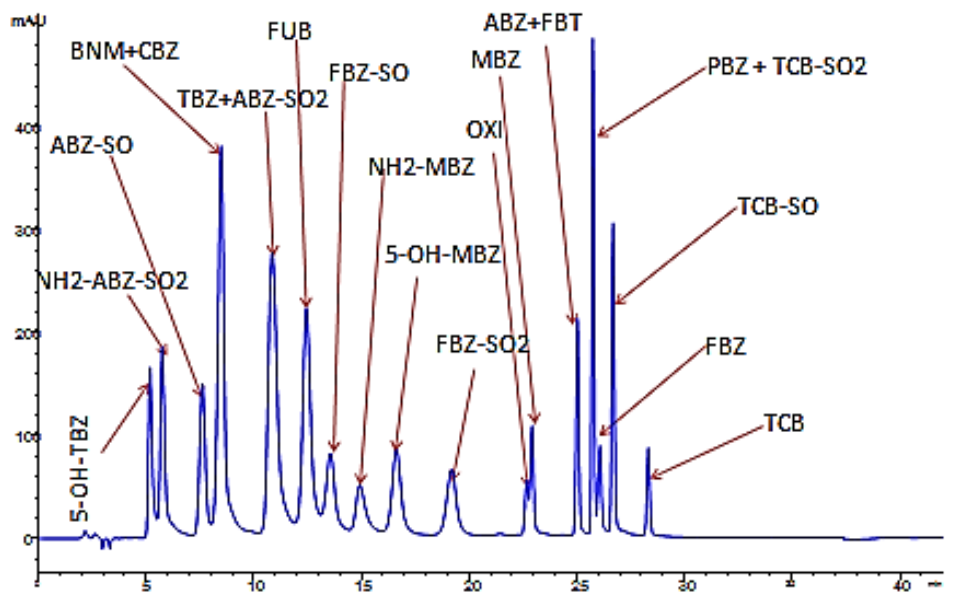

Figura 4: Cromatogramas para la optimización de la velocidad de flujo. 
- Flujo $13 \mu \mathrm{L} / \mathrm{min}$

FLUJO $13 \mu \mathrm{l} / \mathrm{MIN}$

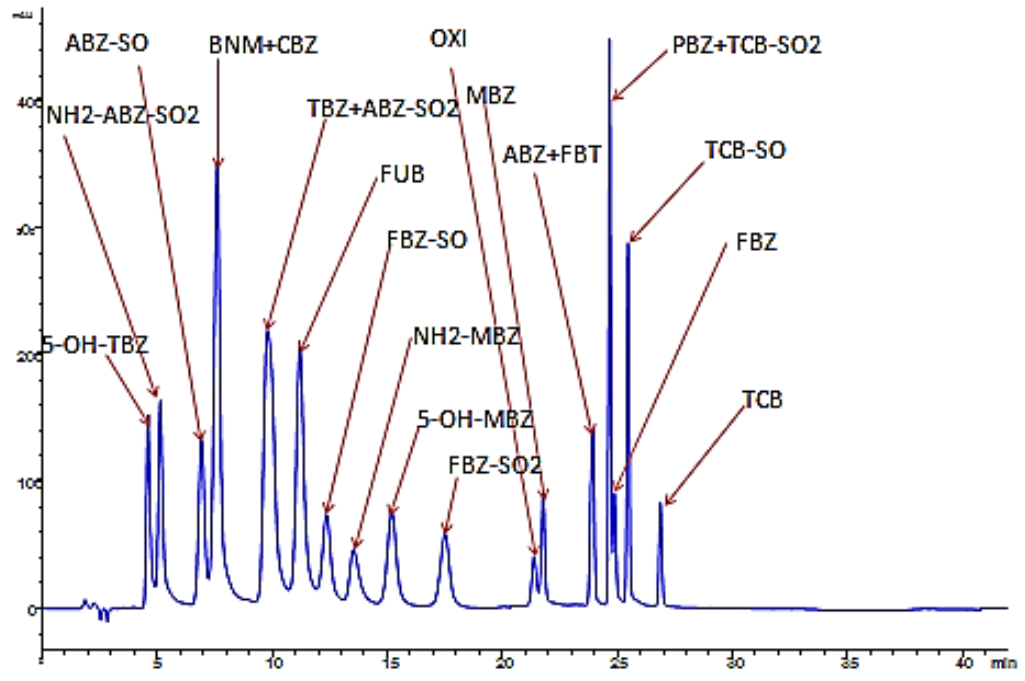

- Flujo $15 \mu \mathrm{L} / \mathrm{min}$

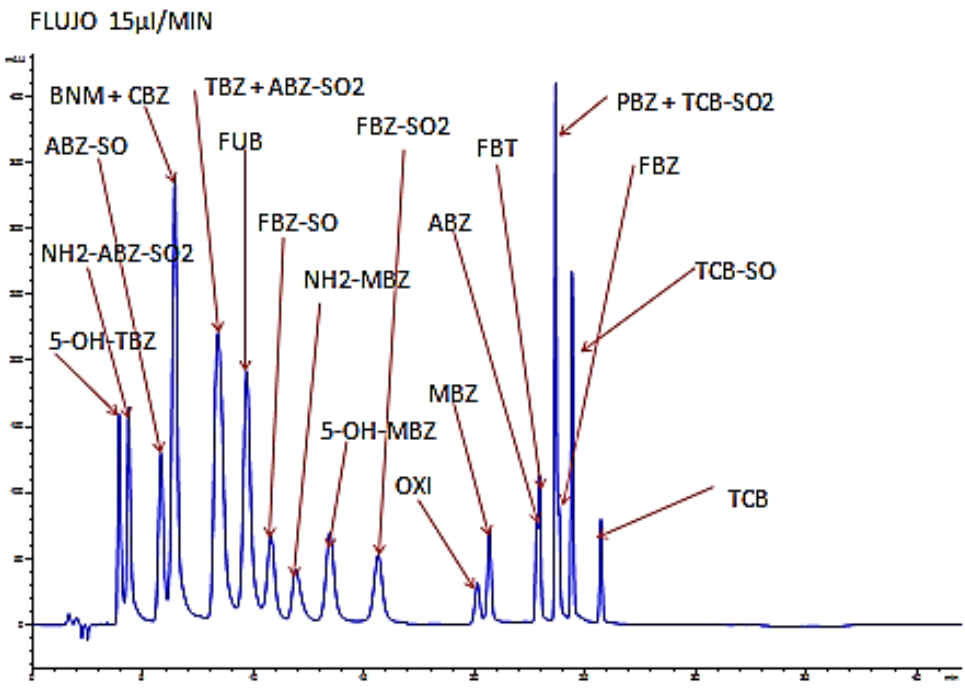

Figura 4: Cromatogramas para la optimización de la velocidad de flujo. 
- Flujo $17 \mu \mathrm{L} / \mathrm{min}$

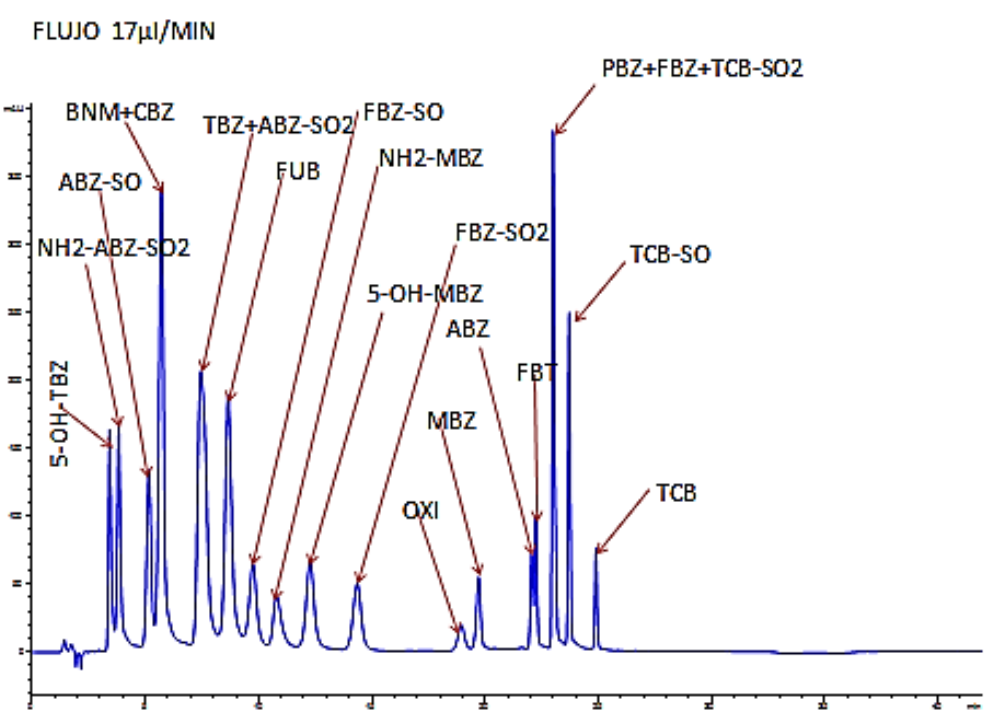

Figura 4: Cromatogramas para la optimización de la velocidad de flujo.

En consecuencia a los resultados, para la elección del flujo las consideraciones que se tuvieron en cuenta fue seleccionar un valor en el que se consiguiera la separación del mayor número de compuestos con mejor resolución y en el caso de descartar algún analito solapado, la prioridad fue la de mantener aquel para el que se resolviesen sus productos de degradación e incluso conservar en la separación el producto de degradación prioritariamente al compuesto original.

Es por ello que en el caso del Tiabendazol (TBZ) y Abendazolsulfona (ABZ-SO ${ }_{2}$ ) se eligió el TBZ puesto que el ABZ ya tenía otros dos metabolitos; en el caso de PBZ y TCB-SO 2 se eligio el metabolito del TCB con el fin que estuviera la familia de TCB; entre $A B Z$ y FBT, nos decantamos por $A B Z$ puesto que es el compuesto del que proceden dos productos de degradación; y entre el MBZ y OXI se elige el MBZ. En el caso del BNM y CBZ aparecen identificados en el mismo pico, pues no se pueden separar.

Tras estas consideraciones, se eligió como flujo óptimo $9 \mu \mathrm{L} / \mathrm{min}$, consiguiendo resoluciones de los analitos superiores a 1.5 .

\subsubsection{Temperatura de la columna}

Posteriormente se procedió a la optimización de la temperatura de la columna. El aumento de la temperatura lleva consigo un aumento de la fluidez de la fase móvil y una menor interacción de los analitos con la fase estacionaria, que provoca una disminución en el tiempo de análisis. 
Se probaron los valores de $T^{a}$ de $20^{\circ}, 30^{\circ}$ y $40^{\circ} \mathrm{C}$. A continuación se muestran los cromatogramas a las $\mathrm{T}^{\mathrm{a}}$ estudiadas.

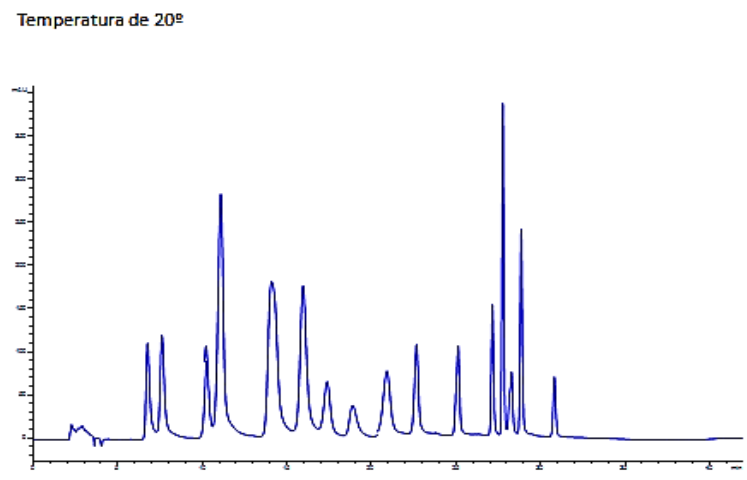

Temperatura de $30^{\circ}$

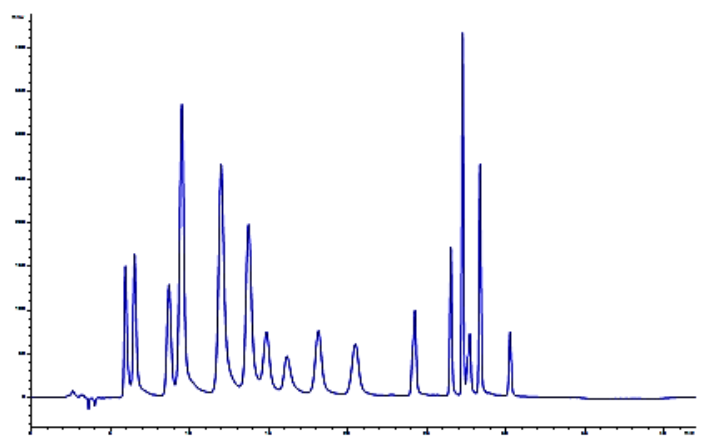

Temperatura de 409

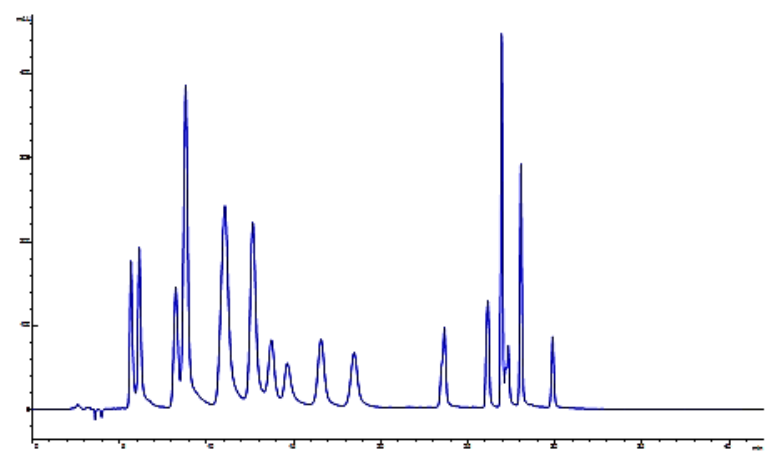

Figura 5: Cromatogramas obtenidos en la optimización de la temperatura de la columna 


\section{TRABAJO FIN DE MÁSTER}

Como se puede observar, al aumentar la temperatura disminuye el tiempo de retención de los analitos pero la resolución empeora. Por lo tanto, mantenemos la temperatura de la columna a $20^{\circ} \mathrm{C}$.

\subsubsection{Disolvente}

Las disoluciones de cada uno de los analitos por separado están preparadas en metanol. A su vez la disolución conjunta preparada a partir de los 17 analitos individualmente que se han decidido estudiar está preparada en agua. Por ello, llevamos a cabo un estudio del disolvente a utilizar para preparar el vial de volumen $1 \mathrm{~mL}$ que más tarde vamos a inyectar en el equipo. A priori se decide realizar estos cambios para que más tarde tengamos el mismo medio de inyección que se usará en el análisis de la muestra tratada. Por ello que se persigue seleccionar un disolvente con un porcentaje de fase orgánica puesto que los analitos se disuelven en metanol.

Se han preparado cuatro viales a una concentración de 50ppb para cada analito, para comprobar la sensibilidad del método y conocer la mínima concentración a utilizar en el calibrado, junto con los resultados del disolvente adecuado. A continuación se muestran los distintos cromatogramas obtenidos al usar diferentes medios de inyección:

- Solvente $\mathrm{H}_{2} \mathrm{O}$.

Disolución a concentración de 50ppb con disolvente $\mathrm{H}_{2} \mathrm{O}$.

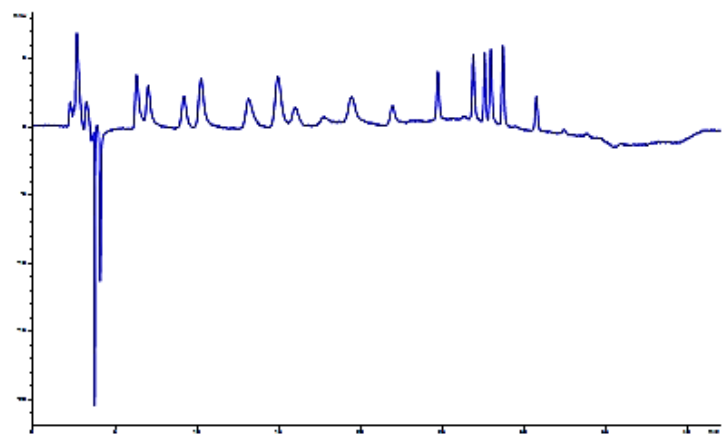

Figura 6: Cromatograma para solvente agua a una concentración de 50ppb. 
Disolución a concentración de 50ppb con disolvente $70 \%$ H2O y 30\% fase orgánica (MeOH:AcCN).

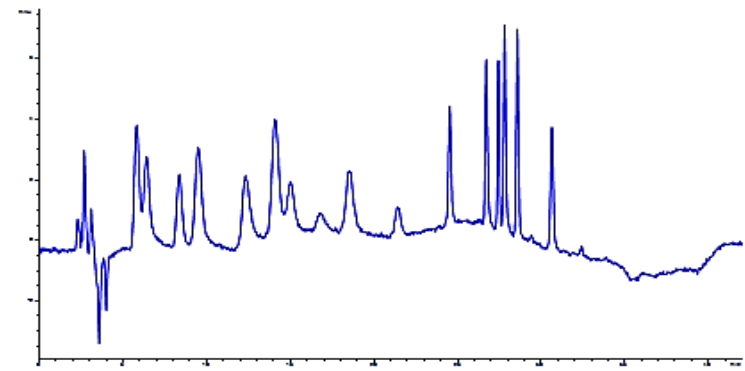

Figura 7: Optimización del disolvente utilizado con $70 \%$ agua y $30 \%$ fase orgánica (MeOH:MeCN) a concentración 50ppb.

Disolución a concentración de 50ppb con disolvente $80 \% \mathrm{H} 20$ y $20 \%$ fase orgánica (MeOH:AcCN).

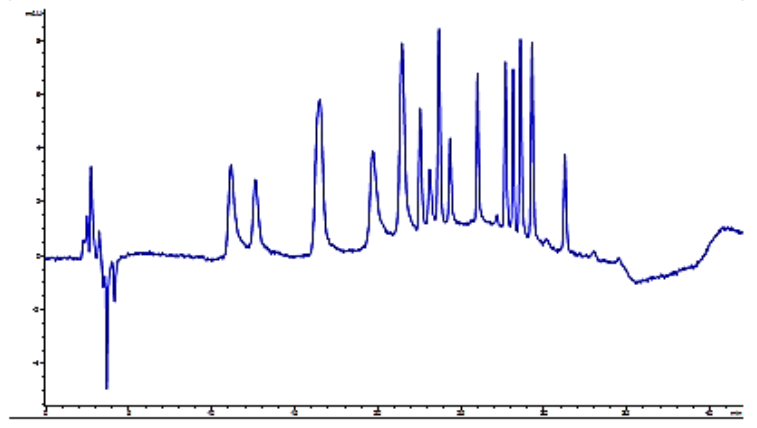

Figura 8: Optimización del disolvente utilizado con $80 \%$ agua y $20 \%$ fase orgánica (MeOH:MeCN) a concentración 50 ppb.

Disolución a concentración de 50ppb con disolvente 70\% Tampón Acetato Amónico y $30 \%$ fase orgánica (MeOH:AcCN).

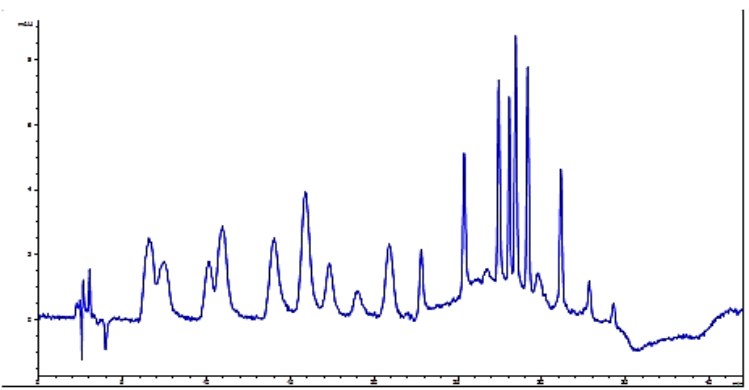

Figura 8: Optimización del disolvente utilizado con $70 \%$ acetato amónico y $30 \%$ fase orgánica (MeOH:MeCN) a concentración 50 ppb. 


\section{TRABAJO FIN DE MÁSTER}

Tras observar los resultados se opta por seleccionar un disolvente de inyección que consiste en 70\% $\mathrm{H}_{2} \mathrm{O}$ y $30 \%$ fase orgánica [MeOH:AcCN (50:50)]. Puesto que cumple con las condiciones que buscamos, con el inconveniente que los dos picos primeros sufren un empeoramiento de la resolución. El siguiente paso es la optimización del volumen de inyección

\subsubsection{Volumen de inyección}

En una primera instancia se observa que el volumen de inyección $(8 \mu \mathrm{L})$ es el máximo permitido por el equipo. Se opta por hacer un estudio de disminución de volumen para comprobar si se mejora la resolución de los picos en las condiciones que tenemos. Esta disminución de volumen lleva consigo que haya una menor cantidad de metanol, que puede que provoque una variación en los tiempos de retención, Para llevar a cabo el estudio se prepara un vial con una disolución mezcla de concentración de 200ppb con disolvente $70 \% \mathrm{H}_{2} \mathrm{O}$ y $30 \% \mathrm{MeOH}: \mathrm{AcCN}$ (50:50). A continuación se muestras los resultados a distintos volúmenes de inyección:
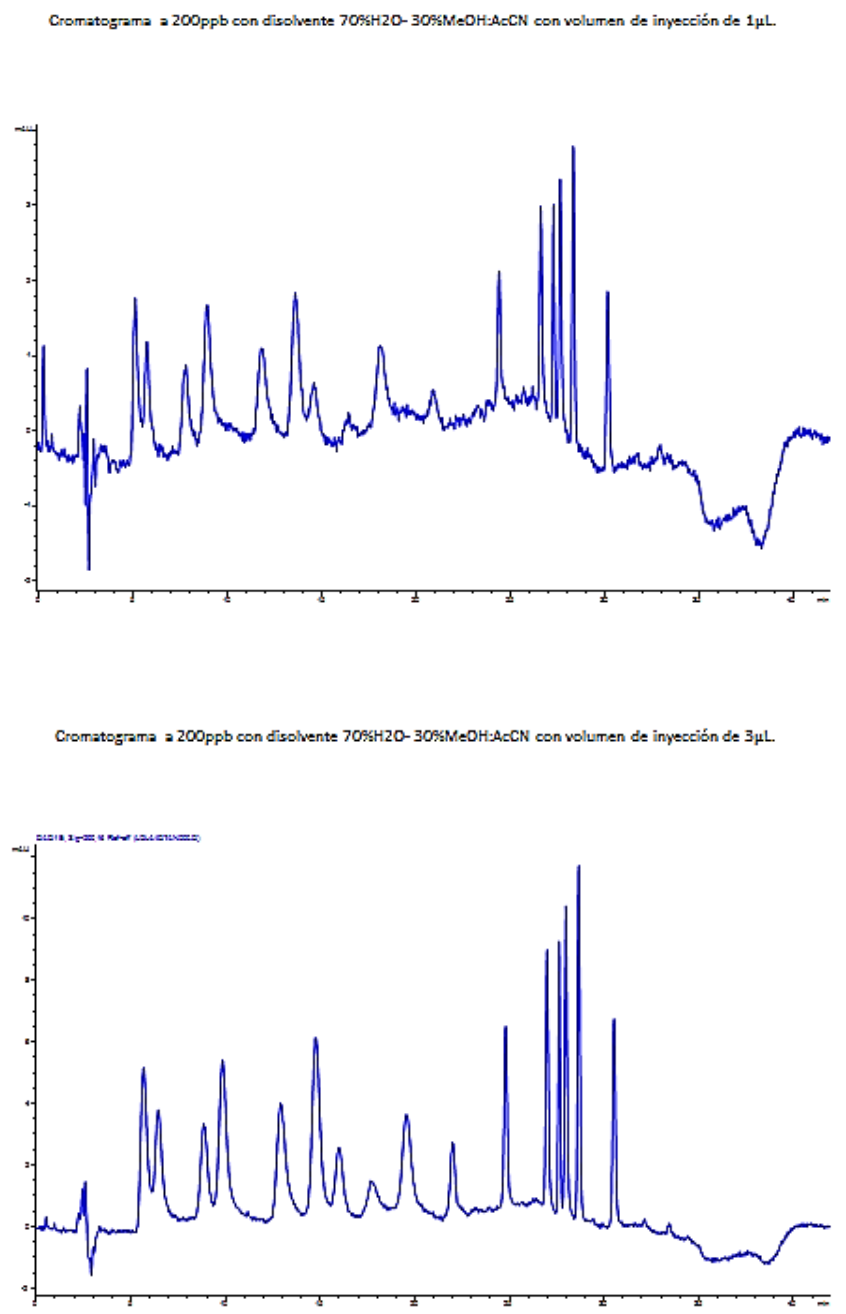

Figura 9: Cromatogramas de la optimización del volumen de inyección. 


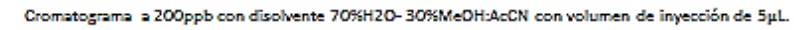

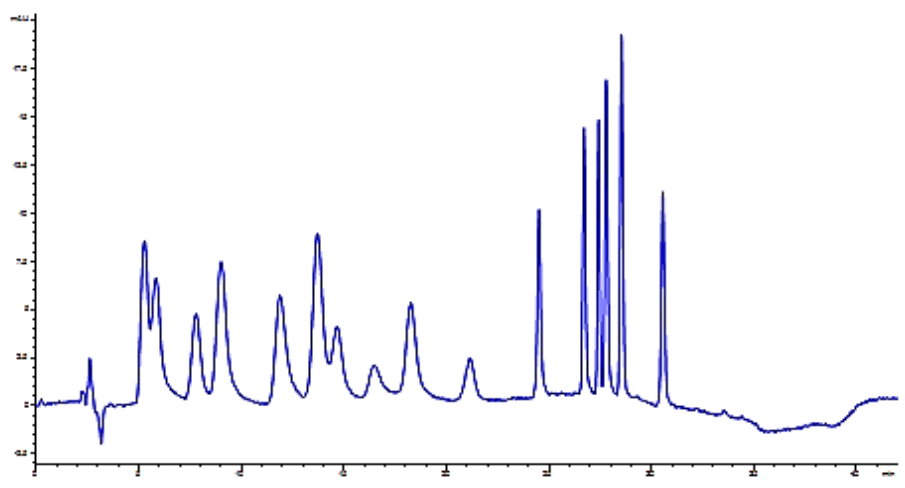

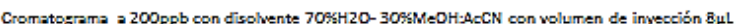

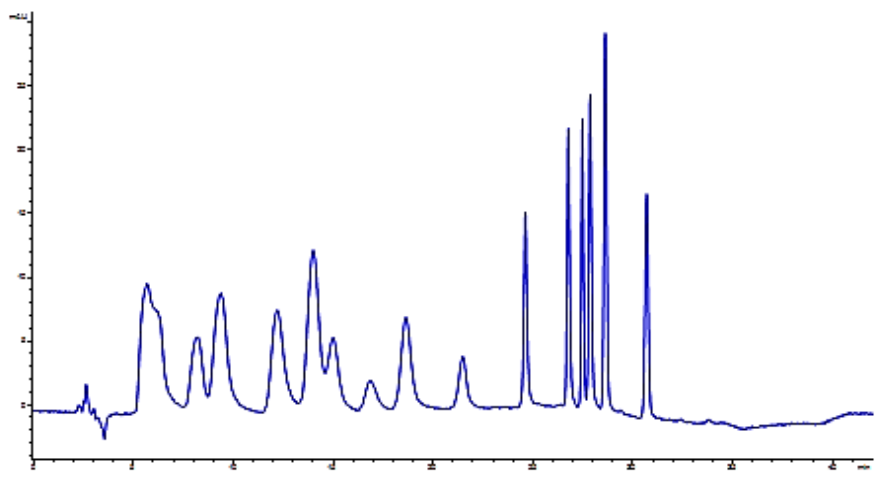

Figura 9: Cromatogramas de la optimización del volumen de inyección.

Tras observar los resultados, optamos por el volumen de inyección de $3 \mu \mathrm{L}$, ya que a volúmenes de inyección superiores la resolución de los picos primeros empeoraba junto con una peor eficacia puesto que los picos se ensanchaban y aparecían a tiempos de retención algo superiores.

Tras la optimización de estos parámetros se lleva a cabo el establecimiento de la recta de calibrado. 


\subsection{RECTA DE CALIBRADO CON PATRONES}

Las condiciones para la realización de la recta de calibrado son:

Volumen inyección

Temperatura columna

Longitud de onda

Flujo

Fase móvil

Gradiente

Disolvente de inyección
$3 \mu \mathrm{L}$

$20^{\circ} \mathrm{C}$

Optima de cada analito

$9 \mu \mathrm{L} / \mathrm{min}$

70\%F.acuosa:Tampón Acetato Amónico

50nM

30\% F. Orgánica: $\mathrm{MeOH}: \mathrm{AcCN}(50: 50)$

el establecido

$70 \% \mathrm{H}_{2} \mathrm{O} 30 \% \mathrm{MeOH}: \mathrm{AcCN}(50: 50)$

Tabla 9: Condiciones óptimas para establecer la recta de calibrado de patrones.

Se preparan 5 disoluciones para llevar a cabo una recta de calibrado con 5 puntos con los 17 analitos a concentraciones de 100, 250, 500, 750 y $1000 \mathrm{ppb}$.

La asignación es fundamental para la identificación y cuantificación de los mismos, puesto que las áreas y alturas de los picos son mayores a dichas longitudes de onda. En estas longitudes, la intensidad de los picos aumenta, así como su eficacia. Sin embargo no afecta ni al tiempo de retención ni a la resolución. A continuación presentamos una tabla donde asignamos a cada analito su longitud de onda óptima.

A continuación se presentan las rectas de calibrado de los 17 analitos. Debido a que BNM y CBZ se determinan conjuntamente, se obtienen 16 rectas de calibrado. Se establecieron considerando el área de pico como señal analítica en función de la concentración de cada analito.

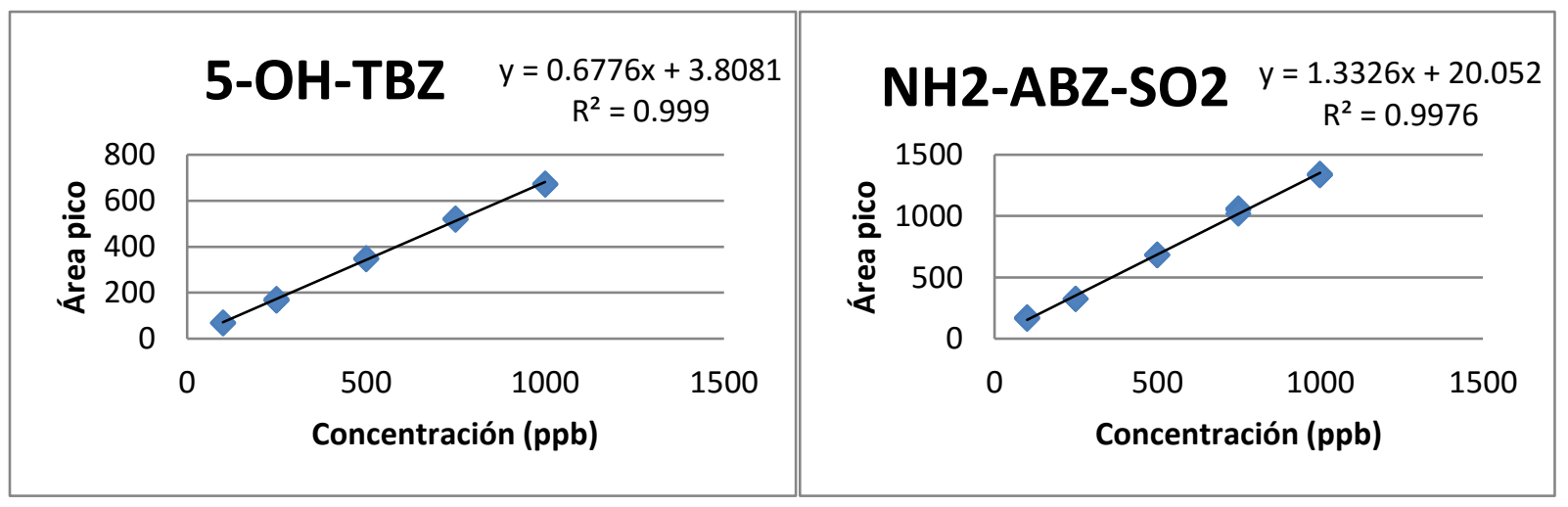

Figura 10: Rectas de calibrado de los compuestos estudiados 

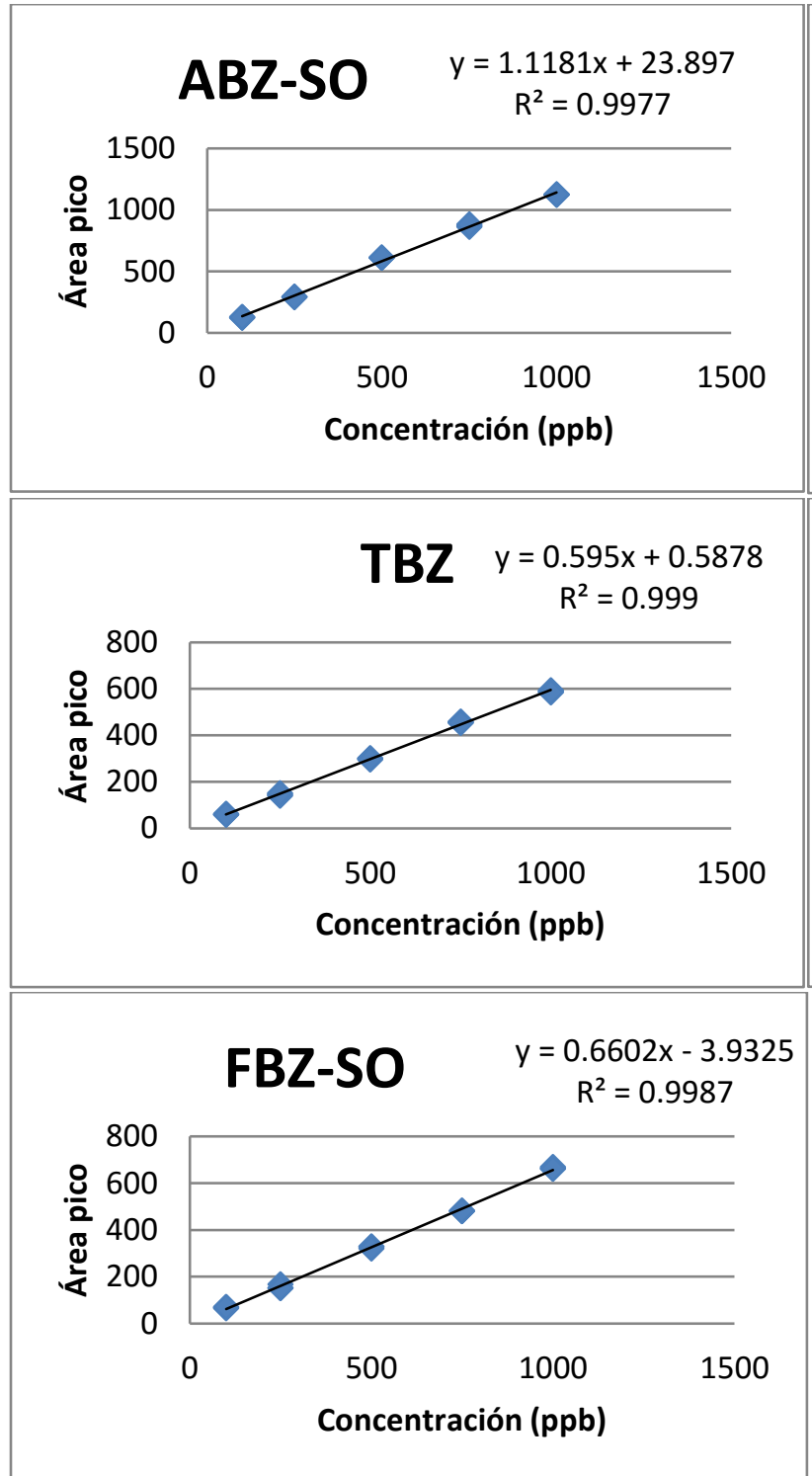

\section{5-OH-MBZ $\quad \begin{gathered}\mathrm{y}=0.4619 x-5.7967 \\ \mathrm{R}^{2}=0.9987\end{gathered}$}

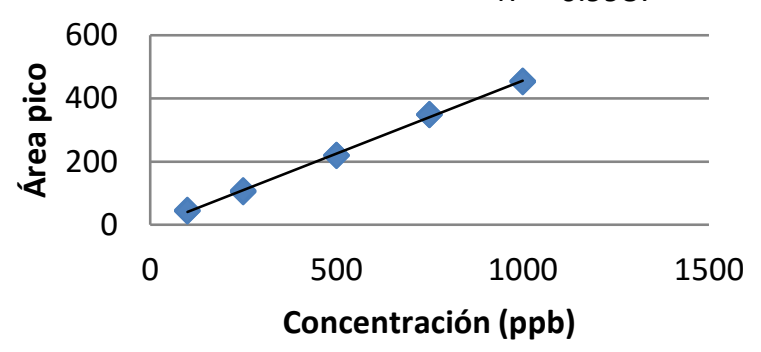

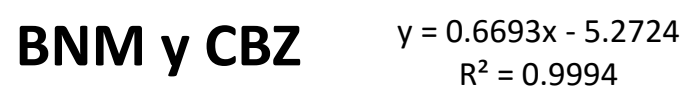
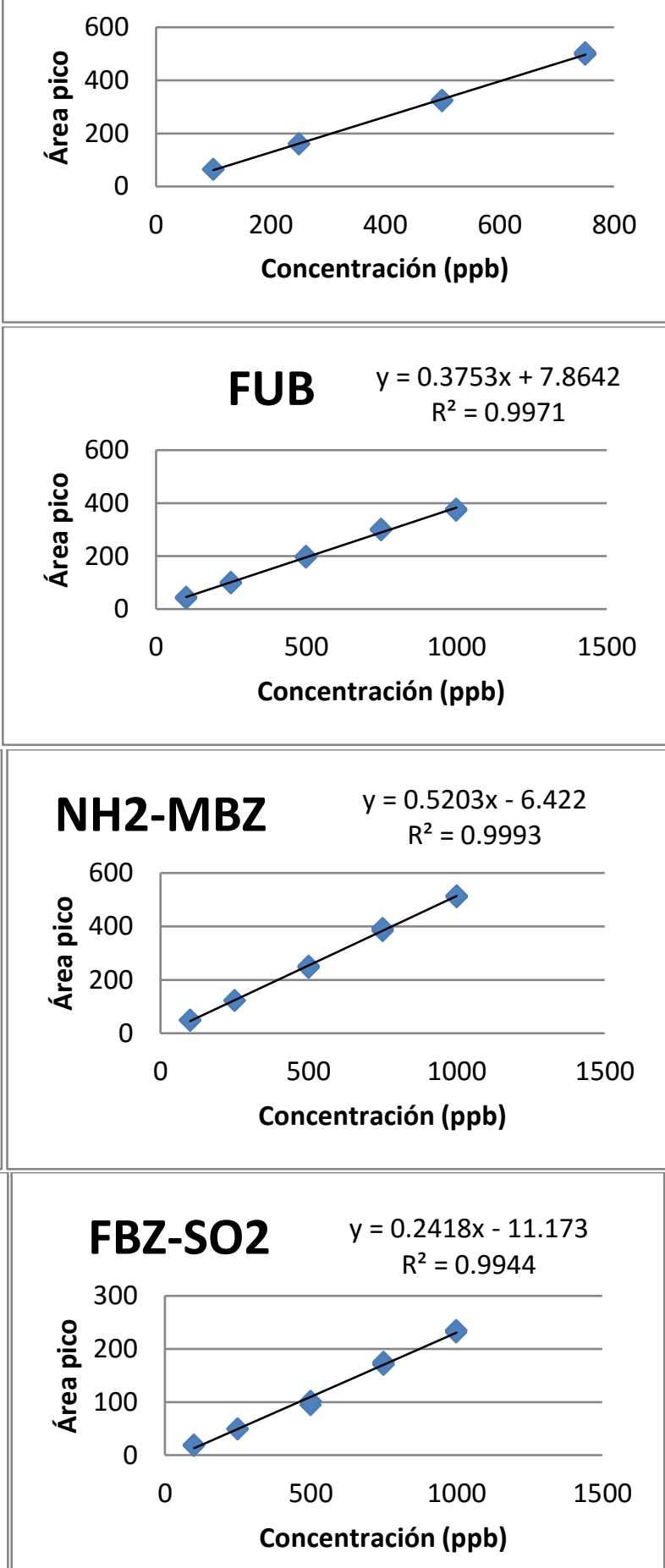

Figura 10: Rectas de calibrado de los compuestos estudiados. 
PROPUESTA DE UNA NUEVA METODOLOGÍA PARA EL CONTROL DE RESIDUOS DE BENZIMIDAZOLES MEDIANTE CROMATOGRAFÍA DE LÍQUIDOS CAPILAR

TRABAJO FIN DE MÁSTER

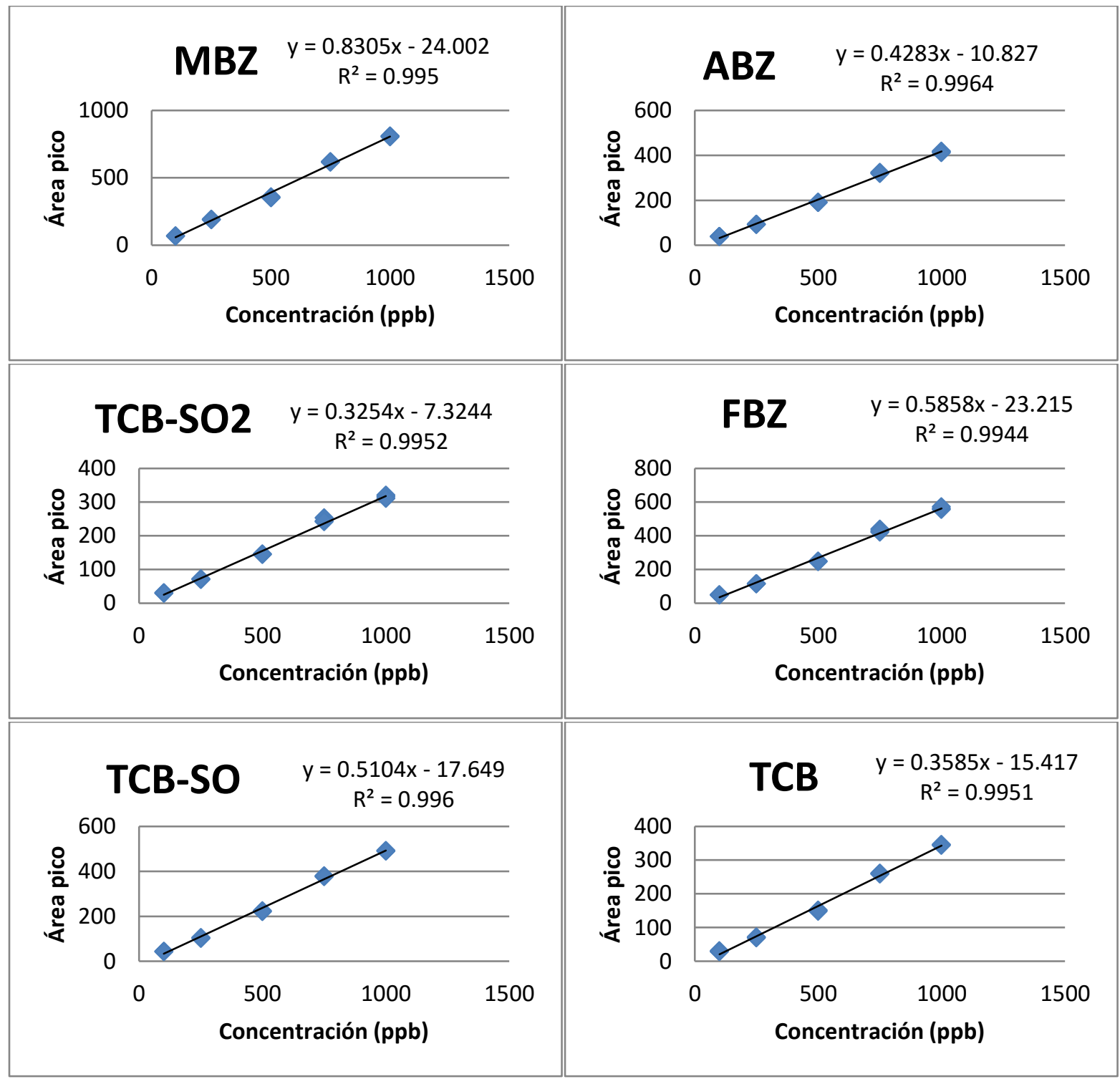

Figura 10: Rectas de calibrado de los compuestos estudiados. 
Las rectas de calibrado están obtenidas adquiriendo las señales longitud de onda óptimas de cada analito, donde se presenta su máximo de adsorción.

\begin{tabular}{|c|c|}
\hline ANALITO & LONGITUD ONDA ÓPTIMA (nm) \\
\hline 5-OH-TBZ & 225 \\
\hline NH2-ABZ-SO2 & 225 \\
\hline ABZ-SO & 225 \\
\hline $\mathrm{BNM}+\mathrm{CBZ}$ & 290 \\
\hline TBZ & 290 \\
\hline FUB & 250 \\
\hline FBZ-SO & 225 \\
\hline NH2-MBZ & 250 \\
\hline 5-OH_MBZ & 290 \\
\hline FBZ-SO2 & 290 \\
\hline MBZ & 250 \\
\hline$A B Z$ & 290 \\
\hline TCB-SO2 & 290 \\
\hline FBZ & 290 \\
\hline TCB-SO & 290 \\
\hline TCB & 290 \\
\hline
\end{tabular}

Tabla 9: Presentacion de las longitudes de onda optima para cada benzimidazol

\subsubsection{Parámetros estadísticos}

Una vez representadas las rectas de calibrado, en la siguiente tabla se muestra la ecuación de la recta y el coeficiente de determinación (R2). Estos parámetros se han calculado mediante regresión lineal por mínimos cuadrado. En la tabla inferior se muestras los datos obtenidos para los 17 benzimidazoles. 
TRABAJO FIN DE MÁSTER

\begin{tabular}{|c|c|c|}
\hline Analito & Ecuación de la recta & $R^{2}$ \\
\hline 5-OH-TBZ & $y=0,6776 x+3,8081$ & 0.999 \\
\hline NH2-ABZ-SO2 & $y=1,3326 x+20,052$ & 0.998 \\
\hline ABZ-SO & $y=1,1181 x+23,897$ & 0.999 \\
\hline $\mathrm{BNM}+\mathrm{CBZ}$ & $y=0,6693 x-5,2724$ & 0.999 \\
\hline TBZ & $y=0,595 x+0,5878$ & 0.999 \\
\hline FUB & $y=0,3753 x+7,8642$ & 0.998 \\
\hline FBZ-SO & $y=0,6602 x-3,9325$ & 0.999 \\
\hline NH2-MBZ & $y=0,5203 x-6,422$ & 0.999 \\
\hline 5-OH_MBZ & $y=0,4619 x-5,7967$ & 0.999 \\
\hline FBZ-SO2 & $y=0,2418 x-11,173$ & 0.994 \\
\hline MBZ & $y=0,8305 x-24,002$ & 0.995 \\
\hline$A B Z$ & $y=0,4283 x-10,827$ & 0.996 \\
\hline TCB-SO2 & $y=0,3254 x-7,3244$ & 0.995 \\
\hline FBZ & $y=0,5858 x-23,215$ & 0.994 \\
\hline TCB-SO & $y=0,5104 x-17,649$ & 0.996 \\
\hline TCB & $y=0,3585 x-15,417$ & 0.995 \\
\hline
\end{tabular}

Tabla 10: Parámetros estadísticos del calibrado de patrones de los 17 benzimidazoles

Parámetros de calidad

A continuación se muestras los parámetros de calidad instrumentales del método, límite de detección (LD) y límite de cuantificación (LQ) y precisión.

Los LDs y LQs se han considerado una relación señal/ruido (S/R) de 3 veces para asignar los LDs y de 10 veces para los LQs. Para la determinación de la relación señal/ruido, el ruido ha sido determinado a la longitud de onda óptima para cada analito en el cromatograma obtenido a una concentración de 100 ppb. Los parámetros de calidad de muestran a continuación:

Tabla 11: LDs y LQs obtenidos en el calibrado de patrones de los 17 benzimidazoles.

\begin{tabular}{|ccccc|}
\hline Analito & $\begin{array}{c}\text { Altura } \\
\text { pico }\end{array}$ & señal/ruido & LD & LQ \\
\hline 5-OH-TBZ & 4,4 & 5,7 & 17,0 & 56,8 \\
\hline NH2-ABZ-SO2 & 7,9 & 3,2 & 9,5 & 31,6 \\
\hline ABZ-SO & 5,2 & 3,5 & 10,4 & 34,6 \\
\hline BNM+CBZ & 2,8 & 5,4 & 16,1 & 53,6 \\
\hline TBZ & 1,8 & 8,3 & 25,0 & 83,3 \\
\hline FUB & 1,6 & 11,3 & 33,8 & 112,5 \\
\hline FBZ-SO & 2,3 & 13,0 & 39,1 & 130,4 \\
\hline NH2-MBZ & 1,8 & 4,4 & 13,3 & 44,4 \\
\hline 5-OH_MBZ & 1,5 & 10,0 & 30,0 & 100,0 \\
\hline
\end{tabular}


PROPUESTA DE UNA NUEVA METODOLOGÍA PARA EL CONTROL DE RESIDUOS DE BENZIMIDAZOLES MEDIANTE CROMATOGRAFÍA DE LÍQUIDOS CAPILAR

TRABAJO FIN DE MÁSTER

\begin{tabular}{|ccccc|}
\hline FBZ-SO2 & 0,52 & 28,8 & 86,5 & 288,5 \\
\hline MBZ & 5,7 & 1,9 & 5,8 & 19,3 \\
\hline ABZ & 4,1 & 3,7 & 11,0 & 36,6 \\
\hline TCB-SO2 & 3,9 & 3,8 & 11,5 & 38,5 \\
\hline FBZ & 5,3 & 2,8 & 8,5 & 28,3 \\
\hline TCB-SO & 4,8 & 3,1 & 9,4 & 31,3 \\
\hline TCB & 2,6 & 5,8 & 17,3 & 57,7 \\
\hline
\end{tabular}

\section{Parámetros de precisión}

La precisión se ha obtenido calculando la desviación estándar relativa (DER) a partir de medidas de disoluciones patrón mezcla de todos los analitos realizadas a las condiciones óptimas del método. Usando tres niveles de concentración diferentes en el mismo día. Se realizan tres réplicas de inyección de cada concentración. En la tabla siguiente se muestran los resultados del estudio.

Tabla 12: Precision del método propuesto.

\begin{tabular}{|cccc|}
\hline & \multicolumn{3}{c|}{ DESVIACIÓN ESTÁNDAR RELATIVA (DER,\%) } \\
\hline ANALITO & \multicolumn{3}{c|}{ CONCENTRACIÓN (ppb) } \\
\hline 5-OH-TBZ & 100 & 500 & 1000 \\
\hline NH2-ABZ-SO2 & 2.19 & 0.75 & 0.51 \\
\hline ABZ-SO & 3.78 & 0.53 & 0.54 \\
\hline BNM+CBZ & 2.89 & 0.15 & 0.24 \\
\hline TBZ & 1.77 & 0.48 & 1.75 \\
\hline FUB & 3.85 & 0.79 & 0.81 \\
\hline FBZ-SO & 6.69 & 1.20 & 0.95 \\
\hline NH2-MBZ & 3.79 & 1.25 & 0.53 \\
\hline 5-OH_MBZ & 3.56 & 1.41 & 0.59 \\
\hline FBZ-SO2 & 5.10 & 0.44 & 0.52 \\
\hline MBZ & 5.49 & 3.87 & 0.95 \\
\hline ABZ & 1.51 & 0.82 & 0.36 \\
\hline & 0.40 & 0.22 & 0.87 \\
\hline
\end{tabular}


TRABAJO FIN DE MÁSTER

\begin{tabular}{|cccc|}
\hline TCB-SO2 & 3.53 & 0.38 & 1.56 \\
\hline FBZ & 2.23 & 0.90 & 1.43 \\
\hline TCB-SO & 1.75 & 1.02 & 0.31 \\
\hline TCB & 5.40 & 1.16 & 0.32 \\
\hline
\end{tabular}

\subsection{TRATAMIENTO DE MUESTRA}

El tratamiento de muestra es una etapa esencial del método analítico en el que la muestra se prepara para extraer los analitos en estudio de la matriz y conseguir la máxima eficacia. Además en nuestro caso decidimos ensayar dos tratamientos de muestra sencillos que requieren de bajos volúmenes de disolventes: la microextracción líquido-líquido dispersiva (DLLME) y la extracción líquido-líquido asistida por sales (SALLE). La matriz seleccionada para el estudio es agua ya que es probable que residuos de estos compuestos puedan llegar a las aguas medioambientales (aguas de piscifactoría, de río, pozo, etc...) procedentes de la práctica agrícola y la actividad humana. Inicialmente el estudio se realizó tomando como muestra agua MiliQ dopada con disolución mezcla de los analitos en estudio para alcanzar una concentración final de 167 ppb.

\section{A) DLLME}

En la DLLME se probaron distintos disolventes extractantes y dispersantes. En una primera instancia se utilizó cloroformo como extractante y metanol como dispersante; sin embargo los dos primeros analitos que son los más polares no aparecen. Por lo que se buscaron ensayos con otros extractantes con valores de logP inferiores (Tabla 13).

\begin{tabular}{cc} 
Extractante & Log P \\
\hline Cloroformo & 1.93 \\
Diclometano & 1.05 \\
Dicloroetileno & 3.07 \\
2-Butanona & 0.47 \\
\hline
\end{tabular}

Tabla 13: Polaridad de diferentes disolventes considerados para la realización del tratamiento de muestra mediante DLLME.

Viendo los valores de polaridad de los disolventes, se optó por realizar el tratamiento de muestra con diclorometano y 2-butanona. EL problema que surgió es que la 2-butanona es menos densa que el agua, además de que la presencia de metanol favorecía su miscibilidad, por lo que desaparecía la separación las fases. Lo dicho antes, tiene su consecuencia en la ausencia de fases tras llevar el tubo falcon a la centrífuga para extraer la fase orgánica, lugar donde se encuentran los analitos que han sido extraídos por el disolvente que utilizamos como extractante. 


\section{TRABAJO FIN DE MÁSTER}

Se hicieron diferentes pruebas en un tubo falcon con diferentes volúmenes de los tres disolventes para comprobar la separación de fases de manera cualitativa, de donde se obtuvo:

- $\quad 5 \mathrm{~mL}$ agua $+5 \mathrm{~mL}$ 2-butanona $\rightarrow$ separación de fases

- $5 \mathrm{~mL}$ agua $+1 \mathrm{~mL} 2$-butanona $\rightarrow$ sin separación de fases

- $5 \mathrm{~mL}$ agua $+3 \mathrm{~mL}$ 2-butanona $\rightarrow$ separación de fases

- $\quad 5 \mathrm{~mL}$ agua $+3 \mathrm{~mL} 2$-butanona $+0.5 \mathrm{~mL}$ metanol $\rightarrow$ sin separación de fases

De las pruebas se puede ver que necesitamos mucha cantidad de extractante, lo que no es conveniente para el secado posterior. Al final, y tras los resultados sólo se consideraron los extractante de cloroformo y diclorometano. En este tratamiento de muestra se ha utilizado como dispersante metanol y como extractante cloroformo en volúmenes [ $\left.\mathrm{CHCl}_{3}: \mathrm{MeOH}(950: 1700, \mu \mathrm{L})\right]$ A continuación de muestran los cromatogramas obtenidos usando estos disolventes extractantes:

Muestra 100pph en agua milia con extractante diclorometano

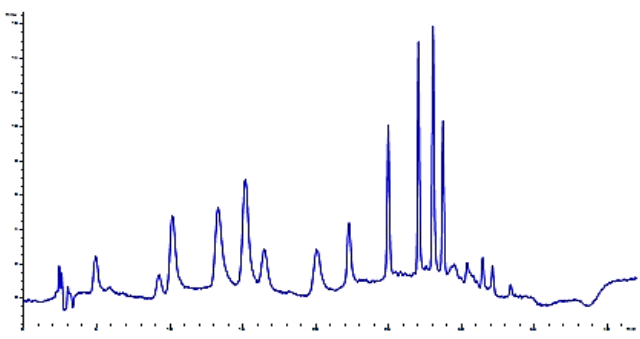

Muestra $100 \mathrm{ppb}$ con agua mili $\mathrm{Q}$, extractantecloroformo

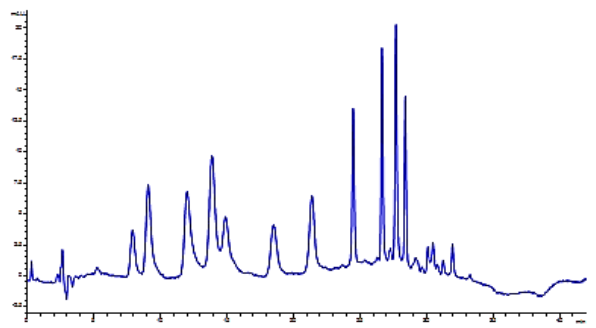

Figura 11: Cromatogramas obtenidos aplicando la DLLME con distintos disolventes extractantes.

Observando los resultados se concluye que ninguno de los dos extractantes nos permite unos resultados adecuados para el tratamiento de muestra; en el caso del diclorometano se pierden los dos primeros analitos, y en el caso del cloroformo se solapan el tercero y cuarto analito. Es por ello que se opta por probar otro tratamiento de muestra (SALLE).

\section{B) SALLE}

El tratamiento de muestra SALLE se realiza para comprobar si se obtienen cromatogramas con una mayor resolución y eficacia que en el tratamiento de muestra DLLME. En nuestro estudio se obtienen mejores resultados. En este tratamiento $\mathrm{MeCN}$ es el disolvente extractante, $\mathrm{NaCl}$ junto con $\mathrm{H}_{2} \mathrm{O}$ se utiliza para la separación de fases. La sal es utilizada para que la separación de fases sea adecuada y determinante y todos los analitos se encuentren en la fase orgánica. La ausencia de sal provoca que a fase orgánica y fase acuosa tenga una separación no definida pudiendo quedar analitos en la fase acuosa. 


\section{TRABAJO FIN DE MÁSTER}

En el tratamiento de muestra basado en la extracción líquido-líquido SALLE los resultados obtenidos son:

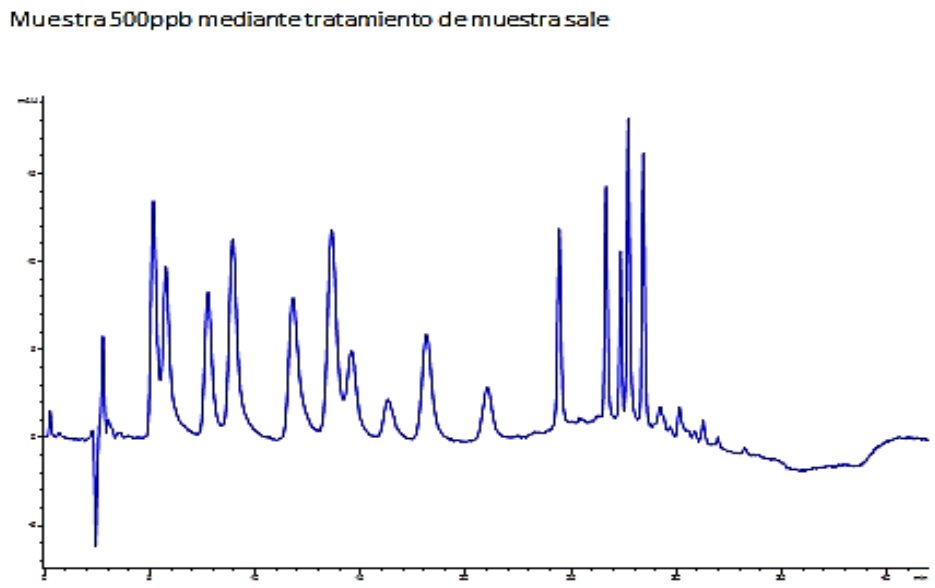

Figura 12: Cromatogramas obtenido al aplicar SALLE a una muestra de agua dopada con 500 ppb.

En comparación con los resultados obtenidos al aplicar la DLLME, los picos mostraban mayores áreas. Con objeto de comprobar la eficacia de este tratamiento se llevó a cabo la inyección directa de una disolución patrón. A la misma concentración (Figura 13)

\footnotetext{
Comparación de cromatogramas de patrón de calibrado a 500 ppb y obten ido mediante el método de tratamiento de muestra de extracción líquido-líquido de sales
}

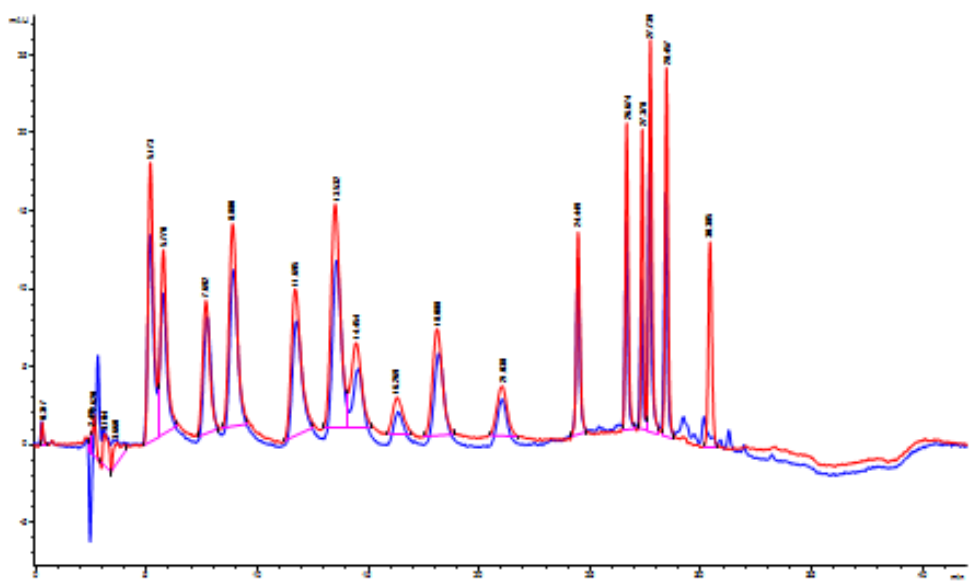

Figura 13: Cromatograma obtenido para una muestra de agua dopada con $500 \mathrm{ppb}$ de analitos y sometida al tratamiento de muestra SALLE (rojo)y una disolución patrón a la misma concentración que se obtiene finalmente en la disolución de inyección. 
Se puede observar como los picos coinciden en tiempo de retención y en áreas, obteniéndose mejores resultados que con la DLLME por lo que se seleccionó este tratamiento de muestra como el más adecuado para extraer los analits de muestras de aguas medioambientales. A continuación se muestras los cromatogramas de los distintos niveles de la recta de calibrado a las distintas concentraciones:

Cromatogramacalibrado muestra a 100ppb

Cromatograma muestracalibrado $250 \mathrm{ppb}$
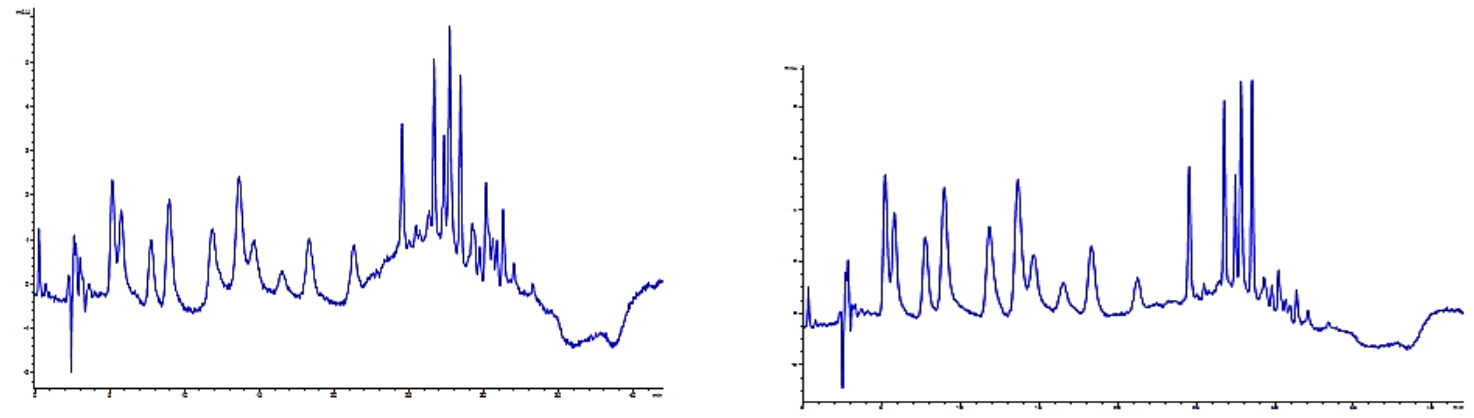

Cromatograma muestracalibrado $750 \mathrm{ppb}$

Cromatograma muestracalibrado $500 \mathrm{ppb}$
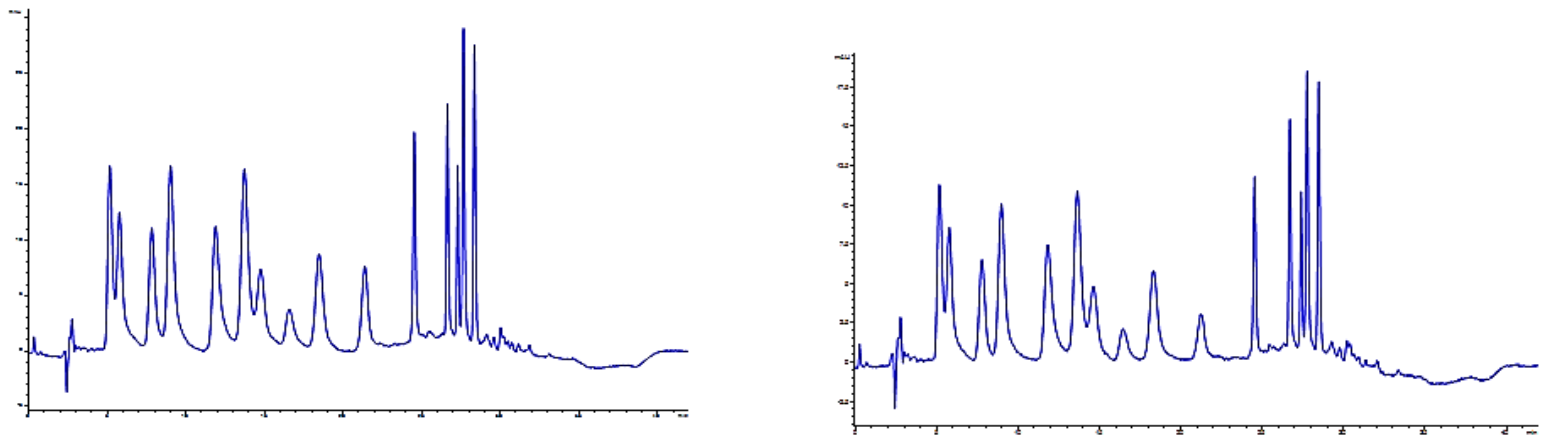

Cromatograma muectracalibrado $1000 \mathrm{ppb}$

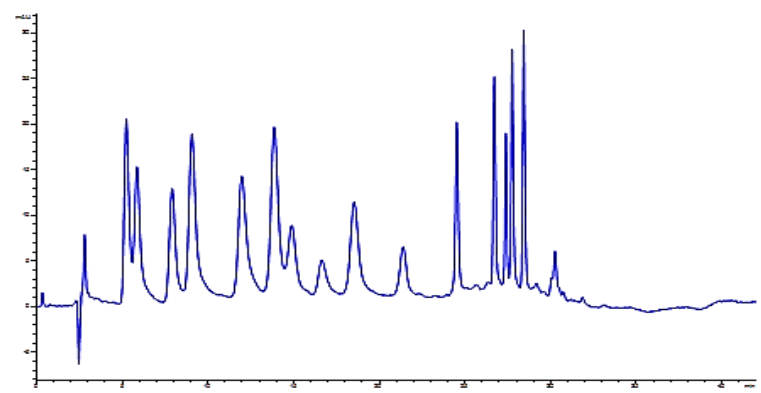

Figura 14: Cromatogramas de muestras tratadas mediante SALLE a diferentes niveles de concentración del calibrado en matriz. 
Se puede ver como al aumentar la concentración de la muestra vemos una mayor eficacia de los picos, pero se observa una disminución de resolución en los primeros picos, en los analitos más polares, resultado de que se eluyen de forma más conjunta.

\subsection{VALIDACIÓN DEL MÉTODO}

Una vez seleccionado el tratamiento de muestra (SALLE) más eficaz para la extracción de los analitos en muestras de aguas medioambientales, se lleva a cabo la validación del método realizando el calibrado en presencia de matriz. Elegimos el mismo rango de concentraciones utilizado en el calibrado de patrones: 66.67, 166.67, 333.33, 500, 666.67 (ppb).

Antes de representar las gráficas de calibrado y los datos obtenidos, así como los diferentes parámetros calculados, se muestras los cromatogramas a cada uno de los niveles de concentración a una longitud de onda de $290 \mathrm{~nm}$. Con esto comprobamos que aparecen todos los benzimidazoles, y en sus tiempos de retención. Además incluimos un blanco de la muestra para saber los interferentes que tendríamos en el cromatograma, tanto del agua de piscifactoría como de los disolventes utilizados en el tratamiento de muestra.

Se prepararán dos viales de la misma concentración y cada vial se pinchará dos veces, teniendo así seis réplicas para cada nivel de concentración. Tras el análisis, no se ha podido identificar el TCB, puesto que en la mayoría de los cromatogramas aparecían picos muy cercanos.

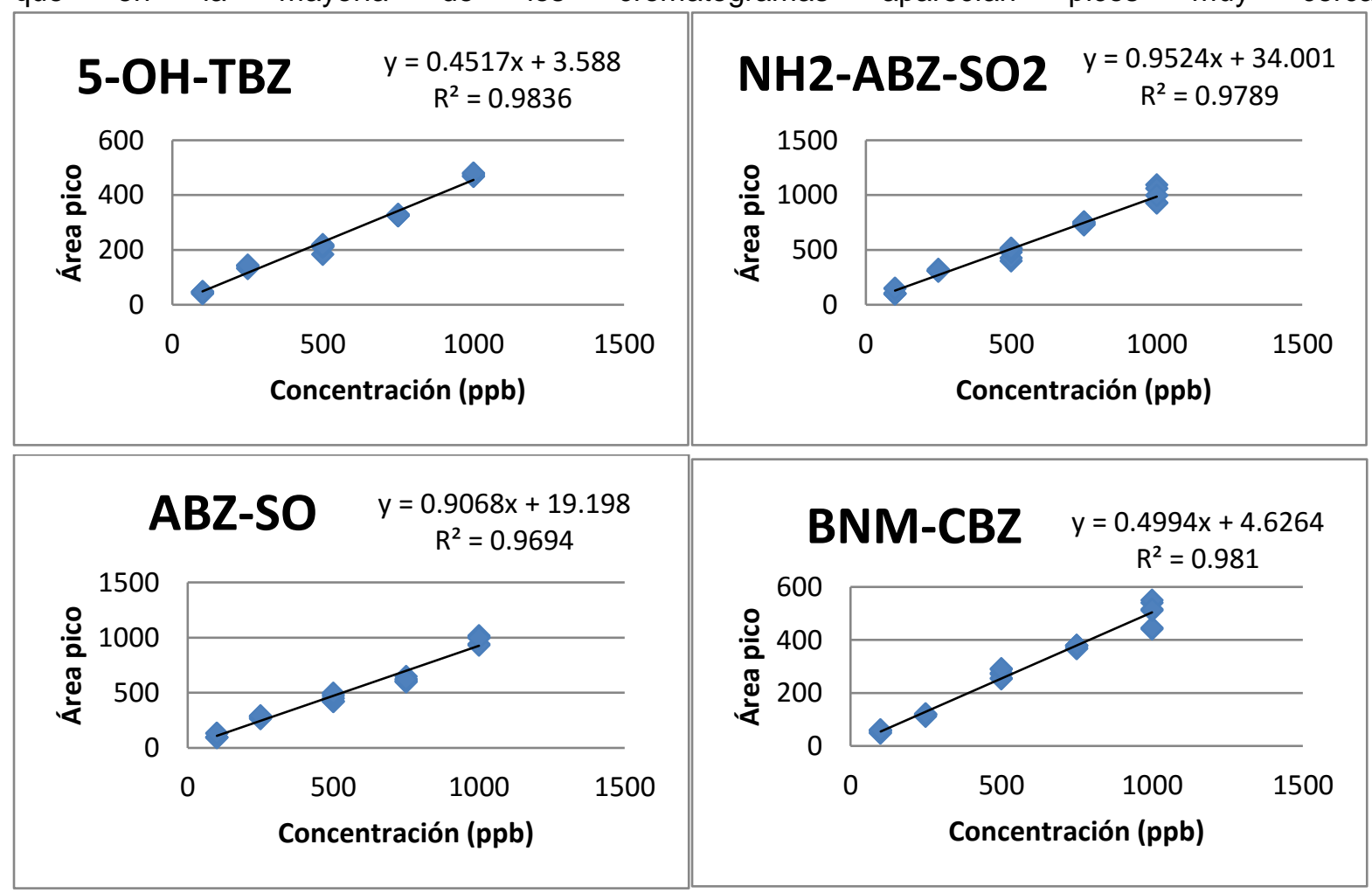

Figura 15: Rectas de calibrado en presencia de matriz (agua de piscifactoría) de la muestra con los analitos estudiados 
PROPUESTA DE UNA NUEVA METODOLOGÍA PARA EL CONTROL DE RESIDUOS DE BENZIMIDAZOLES MEDIANTE CROMATOGRAFÍA DE LÍQUIDOS CAPILAR

TRABAJO FIN DE MÁSTER

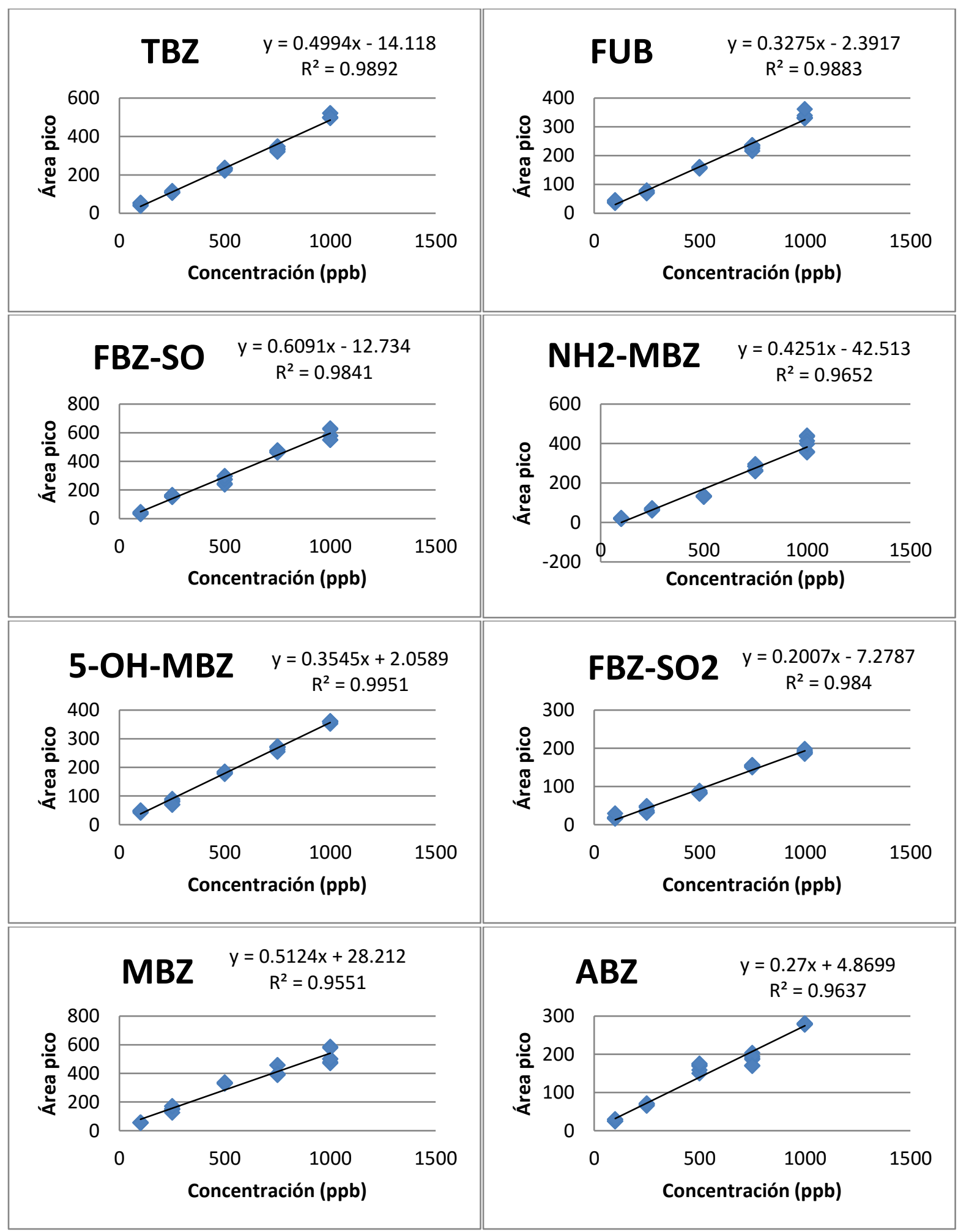

Figura 15: Rectas de calibrado en presencia de matriz (agua de piscifactoría) de la muestra con los analitos estudiados. 
PROPUESTA DE UNA NUEVA METODOLOGÍA PARA EL CONTROL DE RESIDUOS DE BENZIMIDAZOLES MEDIANTE CROMATOGRAFÍA DE LÍQUIDOS CAPILAR

TRABAJO FIN DE MÁSTER
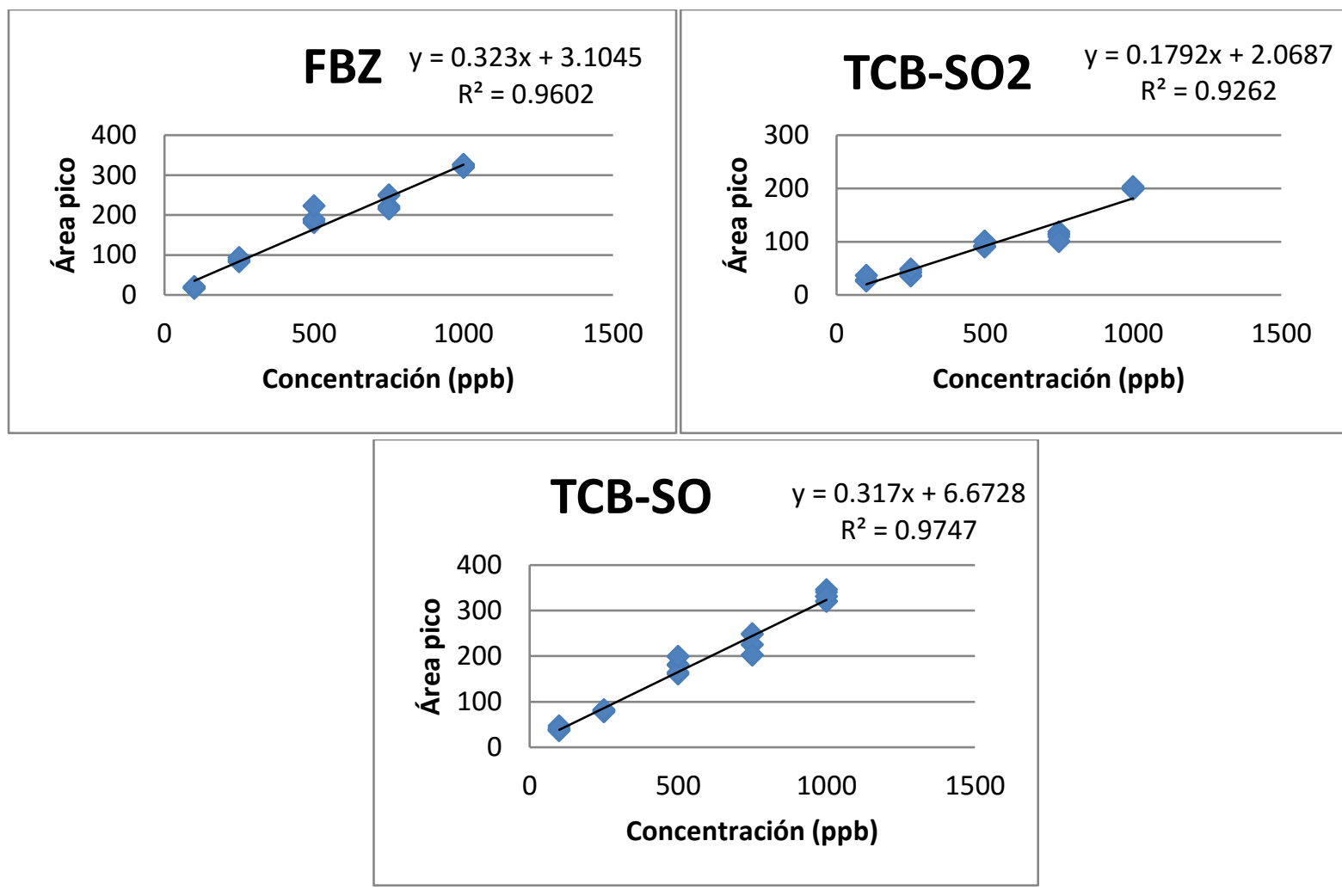

Figura 15: Rectas de calibrado en presencia de matriz (agua de piscifactoría) de la muestra con los analitos estudiados

\subsubsection{Parámetros de calidad}

Los parámetros de calidad obtenidos al realizar el calibrado en presencia de matriz son:

\begin{tabular}{|lllllll|}
\hline Analito & Altura pico & Altura ruido & señal/ruido & LD & LQ & Rango lineal \\
\hline 5-OH-TBZ & 2.8 & 0.1 & 3.6 & 10.7 & 35.7 & $35.7-1000$ \\
\hline NH2-ABZ-SO2 & 5.5 & 0.1 & 1.8 & 5.5 & 18.2 & $18.2-1000$ \\
\hline ABZ-SO & 3.6 & 0.2 & 5.6 & 16.7 & 55.6 & $55.6-1000$ \\
\hline BNM+CBZ & 2 & 1 & 50.0 & 150.0 & 500.0 & $500.0-1000$ \\
\hline TBZ & 1.4 & 0.23 & 16.4 & 49.3 & 164.3 & $164.3-1000$ \\
\hline FUB & 1.5 & 0.1 & 6.7 & 20.0 & 66.7 & $66.7-1000$ \\
\hline FBZ-SO & 2.2 & 0.36 & 16.4 & 49.1 & 163.6 & $163.6-1000$ \\
\hline NH2-MBZ & 0.75 & 0.09 & 12.0 & 36.0 & 120.0 & $120.0-1000$ \\
\hline 5-OH_MBZ & 1.2 & 0.15 & 12.5 & 37.5 & 125.0 & $125.0-1000$ \\
\hline FBZ-SO2 & 0.48 & 0.11 & 22.9 & 68.8 & 229.2 & $229.2-1000$ \\
\hline MBZ & 1.5 & 0.12 & 8.0 & 24.0 & 80.0 & $80.8-1000$ \\
\hline ABZ-SO & 2.4 & 0.3 & 12.5 & 37.5 & 125.0 & $125.0-1000$ \\
\hline TCB-SO2 & 3.4 & 0.25 & 7.4 & 22.1 & 73.5 & $73.5-1000$ \\
\hline
\end{tabular}


PROPUESTA DE UNA NUEVA METODOLOGÍA PARA EL CONTROL DE RESIDUOS DE BENZIMIDAZOLES MEDIANTE CROMATOGRAFÍA DE LÍQUIDOS CAPILAR

TRABAJO FIN DE MÁSTER

\begin{tabular}{|lllllll|}
\hline FBZ & 1.9 & 0.23 & 12.1 & 36.3 & 121.1 & $121.1-1000$ \\
\hline TCB-SO & 3.5 & 0.26 & 7.4 & 22.3 & 74.3 & $74.3-1000$ \\
\hline
\end{tabular}

Tabla 14: Parámetros de calidad a partir del calibrado en presencia de matriz.

Precisión

El estudio de precisión se ha llevado a cabo a concentraciones inferior, media y alta del calibrado en presencia de matriz, sometidos al proceso analítico completo (SALLE-CLC-DAD):

\begin{tabular}{|c|c|c|c|c|c|c|}
\hline \multirow{3}{*}{ Analito } & \multicolumn{6}{|c|}{ CONCENTRACIÓN (ppb) } \\
\hline & \multicolumn{2}{|c|}{100} & \multicolumn{2}{|c|}{500} & \multicolumn{2}{|c|}{1000} \\
\hline & media & RSD & media & RSD & media & RSD \\
\hline 5-OH-TBZ & 45,75 & 7,28 & 213,95 & 6,14 & 475,20 & 1,18 \\
\hline NH2-ABZ-SO2 & 103,70 & 24,45 & 492,90 & 9,90 & 999,20 & 7,40 \\
\hline ABZ-SO & 98,75 & 19,29 & 462,80 & 7,28 & 1000,80 & 3,61 \\
\hline $\mathrm{BNM}+\mathrm{CBZ}$ & 56,60 & 8,92 & 273,15 & 5,85 & 513,60 & 9,08 \\
\hline TBZ & 47,45 & 13,48 & 230,05 & 2,21 & 499,75 & 2,36 \\
\hline FUB & 40,10 & 9,62 & 158,15 & 1,01 & 331,75 & 3,69 \\
\hline FBZ-SO & 39,55 & 12,63 & 273,30 & 8,92 & 578,00 & 6,11 \\
\hline NH2-MBZ & 19,40 & 10,24 & 131,90 & 1,43 & 405,30 & 9,04 \\
\hline 5-OH_MBZ & 46,70 & 5,91 & 180,90 & 1,71 & 359,90 & 0,89 \\
\hline FBZ-SO2 & 17,03 & 30,56 & 83,55 & 3,16 & 190,20 & 2,39 \\
\hline MBZ & 55,60 & 1,86 & 331,15 & 1,01 & 500,75 & 1,01 \\
\hline ABZ-SO & 25,15 & 10,00 & 169,85 & 5,75 & 279,75 & 0,50 \\
\hline TCB-SO2 & 27,60 & 18,80 & 91,75 & 5,59 & 200,40 & 0,88 \\
\hline FBZ & 17,75 & 11,16 & 188,70 & 10,25 & 324,05 & 1,10 \\
\hline TCB-SO & 39,05 & 9,83 & 180,70 & 9,16 & 330,90 & 3,07 \\
\hline
\end{tabular}

Tabla 15: Precisión del método SALLE-CLC-DAD para muestras de agua de piscifactoria a distintos niveles de concentración de los 17 benzimidazoles.

\section{CONCLUSIONES}

Se propone una nueva metodología para el control de residuos de benzimidazoles mediante cromatografía de líquidos capilar. Estos compuestos son antihelmíticos. Se utilizan como agentes antiparasitarios en animales y algunos como plaguicidas en agricultura. La legislación vigente establece unos LMRs para alimentos de origen animal y vegetal que no se pueden sobrepasar para un consumo seguro. Sim embargo en las aguas no existen legislados LMRs aunque debe tenderse a contemplar concentraciones muy bajas en estas muestras.

* El establecimiento del método de cromatografía de líquidos capilar ha requerido la optimización de parámetros que afectan a la calidad de separación. La elección de los valores óptimos se ha 


\section{TRABAJO FIN DE MÁSTER}

realizado con el objetivo de conseguir la máxima resolución y eficacia de los picos en el menor tiempo posible para la separación cromatográfica. Una vez establecidos los parámetros se procedió al establecimiento de la recta de calibrado con patrones.

* Se han evaluado dos tratamientos de muestra para la extracción de los analitos en estudio en muestras de agua de piscifactoría: la DLLME y SALLE, ambos rápidos y de bajo coste, con la mínima utilización de disolventes. Sin embargo SALLE nos otorgaba mejores resultados que DLLME. Mediante la DLLME no se conseguía la extracción de los dos analitos más polares. Sin embargo, mediante la técnica SALLE se consiguió la extracción de todos los analitos de manera reproducible.

* Al realizar la validación del método con muestra de agua de piscifactoría se obtienen resultados satisfactorios, con bajos LDs para los 17 analitos elegidos.

* El método de cromatografía de líquidos capilar propuesto acoplado a SALLE como tratamiento de muestra (SALLE-CLC-DAD) presenta como ventajas su facilidad, rapidez y eficacia, bajo consumo de disolventes y adecuada precisión. Ha demostrado ser una alternativa sencila para el control de residuos de benzimidazoles en aguas medioambientales y su aplicación podría ser ampliada a otras matrices siempre que se combine con los tratamientos de muestra adecuados. 
TRABAJO FIN DE MÁSTER

\section{BIBLIOGRAFÍA}

[1] H.R. Brown, A.R. Matzuk, I.R. Ilves, L.H. Peterson, S.A. Harris, L.H.Sarett, J.R. Egerton, J.J. Yakstis, W.C.Campbell, A.C. Cuckler, J. Am.Chem. Soc. 83 (1961) 1764.

[2] J.P. Brugmann, D.C. Thienpont, I. VanWijngaarden, O.F. Van Parys, V.L.Schuermans, H.L. Lauwers, J. Am. Med. Assoc. 217 (1971) 313.

[3] V.J. Theodorides, J. Chang, C.J. DiCuiolla, G.M. Grass, R.C. Parish, G.C. Scott, Br. Vet. J. 129 (1973) 97.

[4] V.J. Theodorides, R.J. Gyurik, W.D. Kingsbury, R.C. Parish, Experienta 32 (1976) 702.

[5] C. Baeder, H. Bahr, O. Crist, D. Duwel, H. Kellner, R. Kirsch, H. Loewe, E. Schultes, E. Schutz, H. Westen, Experienta 30 (1974) 753.

[6] J.C. Boray, P.D. Crowfoot, M.B. Strong, J.R. Allison, M. Von Schellenaum, M. Orelli, G. Sarasin, Vet. Rec. 112 (1983) 315.

[7] Q.A. McKellar, E.W. Scott, J. Vet. Pharmacol. Ther. 13 (1990) 223.

[8] C.E. Lanusse, R.K. Prichard, Drug Metab. Rev. 12 (1993) 235.

[9] M.M. Nafissi-Varchei, US patent, 4, 406 (1983) 893.

[10] P. Delatour, J. Euzeby, Le Point V'et'er 15 (1983) 63.

[11] W.J. Blanchflower, A. Cannavan, D.G. Kennedy, Analyst 119 (1994)1325.

[12]Libro Blanco sobre Seguridad Alimentaria. Comisión de las Comunicaciones Europeas, 12-1-2000. COM (1999) 719 FINAL

[13] REGLAMENTO 2377/90/CEE del Consejo, de 26 de junio de 1990, por el que se establece un procedimiento comunitario de fijación de los límites máximos de residuos de medicamentos veterinarios en los alimentos de origen animal. DOCE L 224 de 18 de agosto de 1990. Modificado por el Reglamento 1338/2000/CE de la Comisión, 20 de Octubre de 2000. DOCE L 269 de 21 de Octubre de 2000

[14] (H.M. Maykel (2011). Desarrollo de un método MEKC para la determinación de 5-nitroimidazoles en aguas de río.

[15] F. M. Christensen (1998). Pharmaceuticals in the Environment-a Human Risk? Regulatory Toxicology and Pharmacology 28 (3) págs. 212-221.

[16] R.T. Williams (2005). Human Pharmaceuticals: Assessing the Impacts of Aquatic Ecosystems. Florida: Editorial Society of Environmental Toxicology and Chemistry (SETAC)

[17] S.E. Jørgensen, B. Halling-Sørensen (2000). Drugs in the environment. Chemosphere. 40 págs. 691699.

[18] B. Halling-Sørensen, S. Nors Nielsen, P.F. Lanzky, F. Ingerslev, H.C. Holten Lützhøfl, S.E. Jørgensen (1998). Ocurrence, fate and effects of pharmaceutical substances in the environment. Chemosphere. 36 (2), págs. 357-393.

[19]M. Danaher, H.Ruyckb, S.R.H. Crooks, G. Dowling ,M.O'Keeffe, J. Chromatogr.B, 845 (2007) 5

[20]J.A. Bogan, S. Marriner, J. Pharm. Sci. 69 (1980) 422.

[21] R. Halko, C. Padron Sanz, Z. Sosa Ferrera, J.J. Santana Rodríguez, Chromatographia 60 (2004) 151.

[22] R. Halko, C. Padron Sanz, Z. Sosa Ferrera, J.J. Santana Rodríguez, J. AOAC Int., 89 (2006) 1403.

[23] Y. Santaladchaiyakit, S. Srijaranai, J. Sep. Sci. 37 (2014) 3354.

[24] T. Boontongto, Y. Santaladchaiyakit, R. Burakham, Chromatographia (2014) 1557.

[25] Y. Wen, J. Li, F. Yang, W. Zhang, W. Li, C. Liao, L. Chen, Talanta 106 (2013) 119.

[26] D. Chen, Y. Tao, Z. Liu, Z. Liu, L. Huang, Y. Wang, Y. Pan, D. Peng, M. Dai, Z. Yuan, J. Chromatogr. B 878 (2010) 2928.

[27] X. Xia, Y. Dong, P. Luo, X. Wang, X. Li, S. Ding, J. Shen, J. Chromatogr. B 878 (2010) 3174.

[28] A. Martínez-Villalba, E. Moyano, M.T. Galceran, J. Chromatogr. A 1313 (2013) 119.

[29] B. Guo, Z. Huang, M. Wang, X. Wang, Y. Zhang, B. Chen, Y. Li, H. Yan, S. Yao, J. Chromatogr. A 1217 (2010) 4796. 
[30] L.C. Soliman, K.K. Donkor, J. Environ. Sci. Heal. Part B 49 (2014) 153.

[31]J. Shen, J. Tong, H. Jiang, Q. Rao, N. Li, L. Guo, S. Ding, J. AOAC Int. 92 (2009) 1009.

[32] X.Z. Hu, M.L. Chen, Q. Gao, Q.W. Yu, Y.Q. Feng, Talanta 89 (2012) 335.

[33] J. Domínguez-Álvarez, M. Mateos-Vivas, D. García-Gómez, E. Rodríguez-Gonzalo, R. CarabiasMartínez, J. Chromatogr. A. 1278 (2013) 166.

[34] M. Rezaee, Y. Assadi, M.R MilaniHosseini, E. Aghaee, F. Ahmadi, S. Berijani, J. Chromatogr. A 1116 (2006) 1.

[35] C.E. Lanusse, R.K. Prichard, Parasitology 49 (1993) 123.

[36] C. Lanusse, R. Prichard, J. Vet. Pharmacol. Ther. 13 (1990) 170.

[37] C. Lanusse, S. Ranjan, R. Prichard, Am. J. Vet. Res. 51 (1990) 1459.

[38] C. Lanusse, C. Trudeau, S. Ranjan, R. Prichard, J. Vet. Pharmacol. Ther.14 (1991) 101.

[39] Q.A. McKellar, E.W. Scott, J. Vet. Pharmacol. Ther. 13 (1990) 223.

[40] P. Delatour, R. Parish, in: A.G. Rico (Ed.), Drug Residues in Animals, Academic Press Inc., Orlando, FL, 1989, p. 175.

[41] L.C. Davidse, Ann. Rev. Phytopathol. 24 (1986) 43.

[42] D. Gottschall, V. Theodorides, R. Wang, Parasitol. Today 6 (1990) 118.

[43] Residues of some veterinary drugs in animals and foods. Monographs prepared

[44] R.K. Prichard, Int. J. Parasitol. 3 (1973) 409.

[45] E. Lacey, J.H. Gill, Acta Trop. 56 (1994) 245.

[46] E.M. Bennett, C. Bryant, Mol. Biochem. Parasitol. 10 (1984) 335.

[47] E. Lacey, Int. J. Parasitol. 18 (1988) 885.

[48] P. Kohler, Int. J. Parasitol. 31 (2001) 336.

[49] D.L. Sackett, J.K. Varma, Biochemistry 32 (1993) 13560.

[50] M. Lapras, J.P. Deschanel, P. Delatour, J. Castellu, H. Lombard, Bull.

[51] P. Delatour, R. Parish, in: A.G. Rico (Ed.), Drug Residues in Animals, Academic Press Inc., Orlando, FL, 1989, p. 175.

[52] J.A. Bogan, S.E. Marriner, in: M. Fock (Ed.), Albendazole in helminthiasis.

Royal Society of Medicine International Congress and Symposium Series No. 61. London, Royal Society of Medicine, 1984, p. 13.

[53] P. Delatour, Vet. Res. Commun. 7 (1982) 125.

[54] P. Delatour,M. Dandon, F. Garnier, E. Benoit, Ann. Rech. Vet. 13 (1982) 163.

[55] H. Van Den Bossche, F. Rochet, C. Horig, Adv. Pharmacol. Chemother.19 (1982) 67.

[56] P. Delatour, G. Lorgue, D. Courtot, M. Lapras, Rec. Med. Vet. 152 (1976) 467.

[57] V.J. Theodorides, C.J. DiCuillo, T. Nawalinski, C.R. Miller, J.R. Murphy, J.F. Freeman, J.C. Killeen, W.R. Knapp, Am. J. Vet. Res. 38 (1977) 809.

[58] P. Delatour, R. Parish, in: A.G. Rico (Ed.), Drug Residues in Animals, Academic Press Inc., Orlando, FL, 1989, p. 175.

[59] C.E. Lanusse, R.K. Prichard, Drug Metab. Rev. 12 (1993) 235.

[60] C.A.J. Hajee, N. Haagsma, J. AOAC Int. 79 (1996) 645.

[61] M. Hiemstra, J.A. Joosten, A. De Kok, J. AOAC Int. 5 (1995) 1267.

[62] E.C. McManus, F.V.Washko, D.J. Tocco, Am. J. Vet. Res. 27 (1996) 849.

[63] A. Negro, M.L. Alvarez-Bujidos, A.I. Ortiz, J.C. Cubria, R. Mendez, D. Ordonez, J. Chromatogr. 576 (1992) 135.

[64] M. Danaher, PhD Thesis, Univeristy College Cork, Cork, 2003.

[65] N. Arroyo-Manzanares, A.M. Garcia-Campa na, L. Gamiz-Gracia, Anal. Bioanal. Chem. 401 (2011) 2987. 
[66] N. Arroyo-Manzanares, L. Gamiz-Gracia, A.M. Garcia-Campa na, Food Chem. 135 (2012) 368.

[67] L. Campone, A. Piccinelli, L. Rastrelli, Anal. Bioanal. Chem. 399 (2010) 1279.

[68] L. Campone, A.L. Piccinelli, R. Celano, L. Rastrelli, J. Chromatogr. A 1218 (2011)7648.

[69] Arroyo Mazanares N., Huertas-Pérez J.F., Gámiz García L., García Campaña A.M. A new approach in sample treatment combined with UHPLC-MS/MS for the determination of multiclass mycotoxins in edible nuts and sedes. 2013 p. 2

[70] C. Bosch-Ojeda,F.Sánchez-Rojas,Chromatographia69(2009)1149.

[71] M. Rezaee,Y.Yamini,M.Faraji,J.Chromatogr.A1217(2010)2342.

[72]A.V.Herrera-Herrera,M.Asensio-Ramos,J.Hernández-Borges,M.A.Rodríguez-Delgado,

TrACTrendsAnal.Chem29(2010)728.

[73] A.Zgoła-Grześkowiak, T.Grześkowiak, TrACTrendsAnal.Chem30(2011)

1382.

[74] Lara francisco J., García-Campaña Ana M., Moreno-González D., Megersa N., del Olmo-Iruela M. Salting-out assisted liquidliquid extraction combined withc apillary HPLC for the determination of sulfonylurea herbicides in environmental wáter and banana juices amples.(2014) p. 3

[75] Yingying Wena,c, JinhuaLi a, FangfangYang a,b, WeiweiZhang a, WeiranLi a, ChunyangLiao a, Lingxin Chen a, $\mathrm{n}$ Salting-out assisted liquidliquid extraction with the aid of experimental design for determination of benzimidazole fungicides in high salinity samples by high-performance liquid chromatography.

[75]G.G.Nochea,M.E.F.Laespadab,J.L.P.Pavo'nb,B.M.Corderob,S.M.Lorenzo,J.Chromatogr.A1218(2011)6 240-6247.

[76]Comparison of different sample treatments for the analysis of ochratoxin A in wine by capillary HPLC with laser-induced fluorescence detection. N. Arroyo-Manzanares, A. M. García-Campaña, L. Gámiz-Gracia. Analytical and Bioanalytical Chemistry 401 (2011) 2987-2994.

[77]Comparison of different sample treatments for the analysis of quinolones in milk by capillary-liquid chromatography with laser induced fluorescence detection. M. Lombardo-Agüí, L. Gámiz-Gracia, C. Cruces-Blanco, A.M. García-Campaña. Journal of Chromatography A $1218 \quad$ (2011) 4966-4971. [78]lon-paired extraction of cephalosporins in acetone prior to their analysis by capillary liquid chromatography in environmental water and meat samples. C. Quesada-Molina, M. del Olmo-Iruela, A.M. García-Campaña. Talanta 115 (2013) 943-949. [79]Determination of carbamates at trace levels in water and cucumber by capillary liquid chromatography.

D. Moreno-González, J.F. Huertas-Pérez. L. Gámiz-Gracia, A.M. García-Campaña. International Journal of Environmental Analytical Chemistry $91 \quad$ (2011) 1329-1340. [80]Hollow fiber liquid phase microextraction combined with capillary HPLC for selective determination of six sulfonylurea herbicides in environmental waters. A. Gure, F.J. Lara, N. Megersa, A.M. García-Campaña, M. del Olmo-Iruela. Journal of Separation Science 36 (2013)

3395-3401.

[81]Dispersive liquid-liquid microextraction followed by capillary high-performance liquid chromatography for the determination of six sulfonylurea herbicides in fruit juices. A. Gure, F.J. Lara, N. Megersa, M. del Olmo-Iruela, A.M. García-Campaña. Food Analytical Methods 7 (2014) 1465-1473.

[82]Vortex-assisted ionic liquid dispersive liquid-liquid microextraction for the determination of sulfonylurea herbicides in wine samples by capillary high-performance liquid chromatography. A. Gure, F.J. Lara, A.M. García-Campaña, N. Meguersa, M. del Olmo-Iruela. Food Chemistry 170 (2015) 348-353. 
PROPUESTA DE UNA NUEVA METODOLOGÍA PARA EL CONTROL DE RESIDUOS DE BENZIMIDAZOLES MEDIANTE CROMATOGRAFÍA DE LÍQUIDOS CAPILAR

TRABAJO FIN DE MÁSTER

Página

52 